\title{
Physics of Magnetopause Reconnection: A Study of the Combined Effects of Density Asymmetry, Velocity Shear, and Guide Field
}

\author{
Kentaro G. Tanaka, Masaki Fujimoto, and Iku Shinohara \\ Institute of Space and Astronautical Science, Japan Aerospace Exploration Agency, 3-1-1 Yoshinodai, Chuo-ku, \\ Sagamihara-shi, Kanagawa 252-5210, Japan \\ Correspondence should be addressed to Kentaro G. Tanaka, kentaro.g.tanaka@gmail.com
}

Received 31 May 2010; Revised 7 September 2010; Accepted 21 October 2010

Academic Editor: Rudolf A. Treumann

Copyright ( $) 2010$ Kentaro G. Tanaka et al. This is an open access article distributed under the Creative Commons Attribution License, which permits unrestricted use, distribution, and reproduction in any medium, provided the original work is properly cited.

\begin{abstract}
Magnetopause reconnection would be characterized by the density jump across the current sheet, the flow shear across the boundary, and nonzero guide field. While effects of each of these elements have been studied, the effects arising from the combination of these are still unexplored. Two-dimensional full-particle simulations show that the combination of shear flow and/or guide field with density asymmetry induces the sliding motion of the X-line along the magnetopause. The direction of the $\mathrm{X}$-line motion is controlled either by the ion flow at the X-line when the shear flow effects dominate or by the electron flow at the X-line when the guide field effects dominate. The shear flow effects and the guide field effects may counteract each other in determining the direction of the $\mathrm{X}$-line motion and, in the close proximity of the subsolar region where the flow is slow, the $\mathrm{X}$-line motion can be opposite to the flow direction.
\end{abstract}

\section{Introduction}

Magnetic reconnection drives explosive phenomena in laboratory and astrophysical plasmas. It is one of the most important fundamental processes in the plasma universe (e.g., [1-3]). The magnetic reconnection is associated with a geometrical change in magnetic field structure, namely, the "X-line" formation.

Numerous studies of magnetic reconnection have been carried out assuming symmetric current sheets, which nicely model some of the situations seen in the nature, such as those in the Earth's magnetotail. Sharma et al. [4] recently reviewed the magnetotail reconnection topic. In general, however, the electromagnetic and thermodynamic states across a current sheet are not always symmetric. A good example is seen at the Earth's dayside magnetopause, where there is a significant jump in the plasma properties (e.g., density and temperature) across the current sheet. In-situ observations show that the magnetopause reconnection indeed occurs under an asymmetric condition (e.g., [5-8]). Asymmetric reconnection is also observed in the solar wind (e.g., $[9,10])$ and is expected in astrophysical situations $[11,12]$.

Recently, Borovsky and Hesse [13] used resistive magnetohydrodynamics (MHD) simulations to study reconnection rates as a function of the density asymmetry. Cassak and Shay [14] performed a Sweet-Parker type analysis of the structure of the dissipation region to predict that the stagnation point is shifted towards the magnetospheric side of the X-line. This means that the plasma inflow along the current sheet normal is allowed to cross the $\mathrm{X}$-line from the magnetosheath side to the magnetospheric side. In other words, the $\mathrm{X}$-line site is filled by denser plasma of the magnetosheathorigin flowing towards the lessdense magnetospheric side. They have extended their theory to the Hall-MHD regime [15] to find good agreements between the theory and two-fluid simulation results.

The first step in particle simulations aiming at the magnetopause reconnection is to superimpose a uniform guide field on the symmetric Harris [16] current sheet (e.g., [17-23]). Some simulations [24-26] studied the effects of 
diamagnetic drifts on reconnection by taking into account the asymmetries of both density and guide field intensity. The density asymmetry was also taken into account in the two-dimensional (2D) full-particle simulations utilized to assist data analyses of the magnetopause reconnection events $[27,28]$. Tanaka et al. [27] not only reproduced the features identified by insitu observations but also succeeded in elucidating the electron dynamics associated with magnetopause reconnection, which is not fully resolved by the currently available dataset.

In addition to density asymmetry and nonzero guide field, velocity shear across the magnetopause current sheet is another major item that characterizes the magnetopause reconnection. La Belle-Hammer et al.'s [29] investigated magnetic reconnection in the presence of sheared flow and density asymmetry by $2 \mathrm{D}$ resistive MHD simulations. They showed the X-line to move in the same direction as the magnetosheath flow. The solar wind reconnection under an asymmetric situation with a shear flow studied by Eriksson et al. [9] showed good agreements with the results given by La Belle-Hammer et al. simulation [29].

Density asymmetry, nonzero guide field, and shear flow are the three major items that characterize the magnetopause reconnection situation. While some studies have been performed to see the effects of these elements, a systematic study using full-particle simulations is not available yet. As will be shown, an X-line in motion is one of the key characteristics of the magnetopause reconnection, where the density asymmetry is combined with more than one of the other effects. Then, it is best to retain full description of the X-line physics in the study, namely, to use full-particle simulations.

Regarding the mechanism of the X-line motion along the current sheet, we propose the slanted (from the current sheet normal direction) ion inflow towards the X-line to be one of the mechanisms. Let us outline a simple argument that, in the presence of density asymmetry and shear flow or nonzero guide field, the ion flow at the X-line from the magnetosheath side should be slanted. Here, it should be noted that we are dealing with the ion flow and that only the sign of the guide field component matters in the argument. Unlike in the argument for the electron flow, the nonuniformity of the guide field intensity across the magnetopause (to be more precise, the local current direction right at the $\mathrm{X}$-line) is not considered to be the essential factor in this line of thinking.

The argument using Figure 1 and the simulation runs shown here are performed in the frame where the flow is symmetric across the magnetopause (see (b)). This is the natural choice because the symmetry is recovered as soon as the density is made symmetric. While the magnetosphere rest frame will be used when the discussion is observer oriented, this frame will be used for discussion of the basic physics.

Panel (a) depicts the effect studied by Cassak and Shay [14]. The density asymmetry alone makes the ion stagnation point to be shifted toward the magnetospheric side. In the absence of any other effects, the ion flow from the magnetosheath is along the current sheet normal and does not induce an X-line motion. Panel (b) shows the effects of a shear flow. The ion inflow towards the X-line is now slanted. In the absence of density asymmetry, however, the two inflows meet at the X-line, and the ion flow component along the current sheet at the X-line is cancelled to become zero. Panel (c) shows the situation when both density asymmetry and shear flow are present. Now with the shift of the Xline toward the magnetospheric side, the ions filling the $\mathrm{X}$ line site are those from the magnetosheath inflowing in a slanted manner. The ion flow component along the current sheet is nonzero at the $\mathrm{X}$-line. There is a possibility for an $\mathrm{X}$-line motion to be induced in the same direction as the magnetosheath ion flow. Panel (d) shows that a slanted inflow can be created by the effect of nonzero guide field. While the inflow is essentially normal to the current sheet outside of the current sheet, the flow becomes slanted near the center of it due to the Lorentz force (The same flow pattern is also found in $[18,25]$. When density asymmetry is also present, the combined effects would produce a situation very similar to that depicted in Panel (c), and thus a sliding motion of the X-line.

Regarding the situation where density asymmetry and nonzero guide field are combined, there may well be another explanation. Swisdak et al. $[12,24]$ proposed that it is the electron flow at the $\mathrm{X}$-line that regulates the $\mathrm{X}$-line motion. The cases to be studied here, unlike the ones studied by Swisdak et al. [24], have uniform guide field, and there is no current density arising from inhomogeneity of guide field (i.e., $\partial B_{M} / \partial N=0$. See the coordinates of Figure 1) along the magnetopause current sheet in the initial condition. As the magnetopause reconnection develops under the density asymmetry, however, there generated nonzero gradient of the guide field along the current sheet normal at the X-line, indicating that nonzero current density along the current sheet emerges at the X-line. The current density is sustained by the electron flow at the $\mathrm{X}$-line along the current sheet. That is, when density asymmetry and nonzero guide field are combined, the electron flow at the $\mathrm{X}$-line along the current sheet, which is another candidate to drive the X-line motion, is generated.

The structure of this paper is such that the above two mechanisms for the X-line motion will be tested in a stepby-step manner using full-particle simulations. As will be shown, the systematic survey of the results from the cases that include all the elements characterizing magnetopause reconnection (density asymmetry, shear flow, and nonzero guide field) is useful in elucidating the issue.

\section{Simulation Setup}

We use 2 1/2-dimensional (two spatial dimensions $(N, L)$ and three velocity components $\left(v_{L}, v_{M}, v_{N}\right)$ ) electromagnetic full-particle simulation [30]. The LMN coordinates set the basic framework for magnetopause studies, with the $L$ axis pointing to the direction of the magnetospheric field and the $\mathrm{N}$-axis pointing outward along the magnetopause normal. The Harris-type current sheet is used to ensure that the initial condition is not far from a Vlasov equilibrium. Full details are given below. 


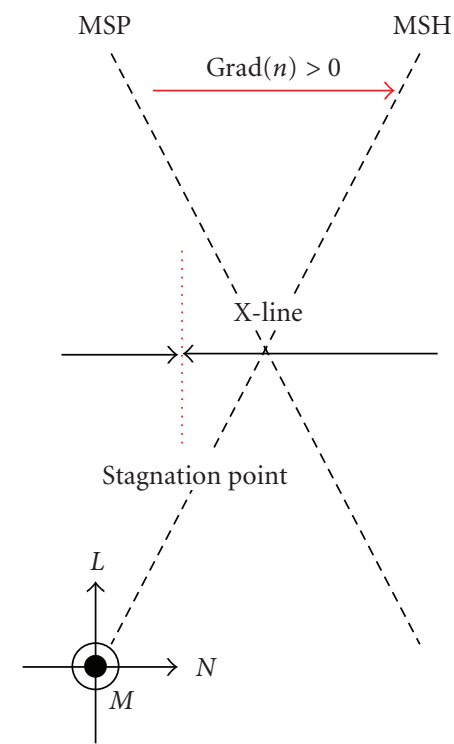

(a) density asymmetry, $V_{\mathrm{MSH}}=0$, $B_{M o}=0$

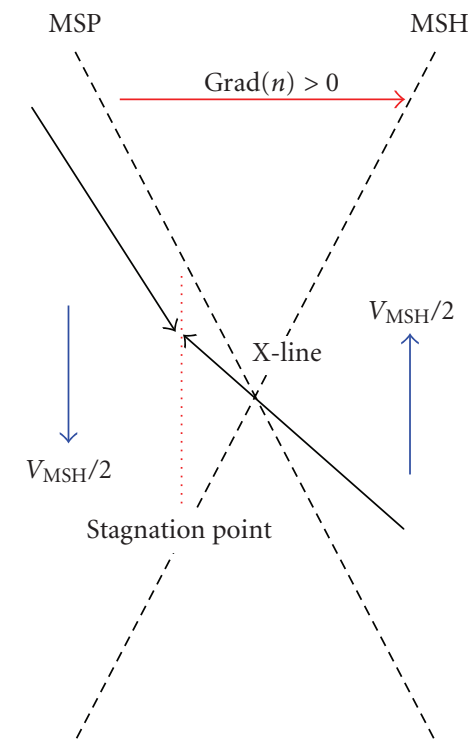

(c) density asymmetry, $V_{\mathrm{MSH}} \neq 0$, $B_{M o}=0$

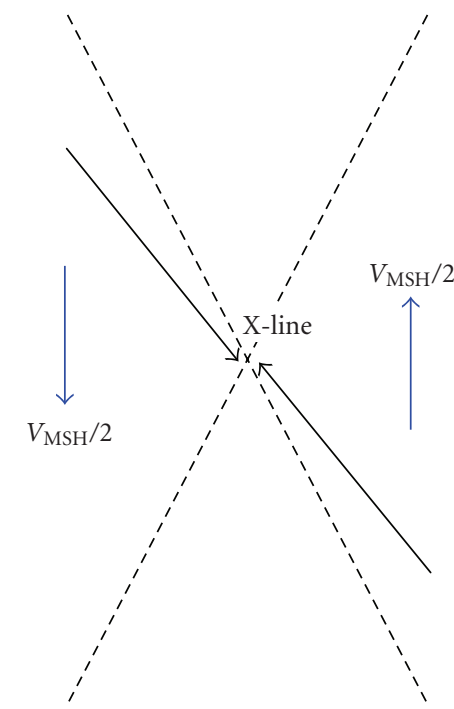

(b) density symmetry, $\quad V_{\mathrm{MSH}} \neq 0$, $B_{M o}=0$

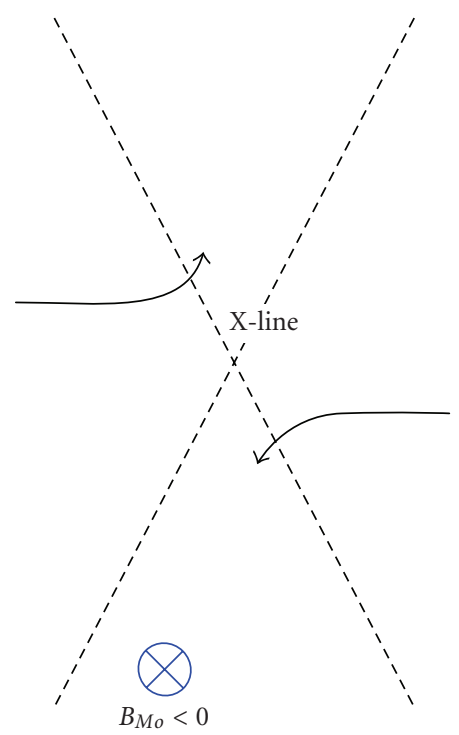

(d) density symmetry, $V_{\mathrm{MSH}}=0$, $B_{M o} \neq 0$

FIGURE 1: A schematic diagram showing why an X-line should move in the magnetopause reconnection situation. (a) Shift of stagnation point towards the magnetospheric side in the presence of density asymmetry. Here, neither a velocity shear nor a guide field is imposed. (b) The effect of a velocity shear in a density symmetric case. The inflow towards the X-line is slanted from the current sheet normal direction. (c) The ion flow pattern at the X-line when density asymmetry and velocity shear are combined. The X-line site is occupied by magnetosheath ions having nonzero component along the current sheet. (d) The effect of nonzero guide field in a density symmetric case. Due to the Lorentz force near the diffusion region, the inflow towards the X-line becomes slanted from the current sheet normal direction.

Two plasma populations are set in the initial condition. One population constitutes the Harris current sheet [16]. The Harris current sheet is expressed as $n_{\mathrm{CS}}=n_{0} / \cosh ^{2}(N / D)$ for the plasma density and as $B_{L}=-B_{0} \tanh (L / D)$ (see Figure 2(f)) for the magnetic field, where $D$ is the half-thickness of the current sheet. The plasma inside the current sheet has the ion-to-electron temperature ratio of $T_{i, \mathrm{cs}}=8 T_{e, \mathrm{cs}}$. The other plasma population is the background plasma having the number density of $n_{\mathrm{BG} 1}=n_{1}\left\{1-1 / \cosh ^{2}(N / D)\right\}$ at $x_{N}>0$ (the magnetosheath side) and $n_{\mathrm{BG} 2}=n_{2}\left\{1-1 / \cosh ^{2}(N / D)\right\}$ at $x_{N}<0$ (the magnetospheric side) (see Figure 2(e)). It is distributed outside the current sheet. In the present paper, the background plasma density is set as $n_{1}=n_{0}$ and $n_{2}=0.2 n_{0}$, respectively. That is, there is a factor of fivedensity difference across the magnetopause current sheet. 


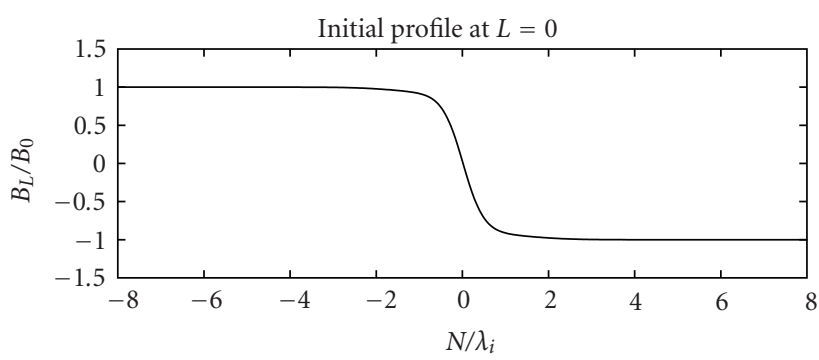

(a)

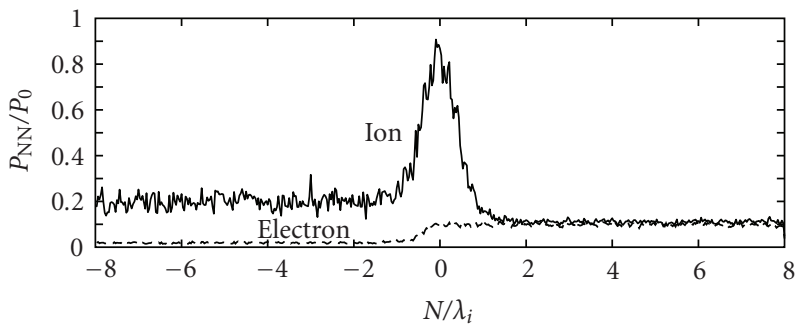

(c)

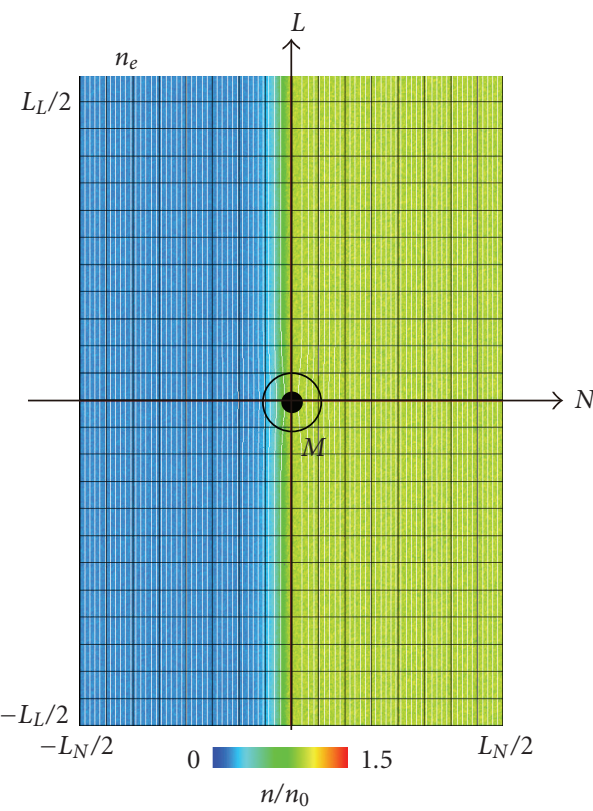

(e)

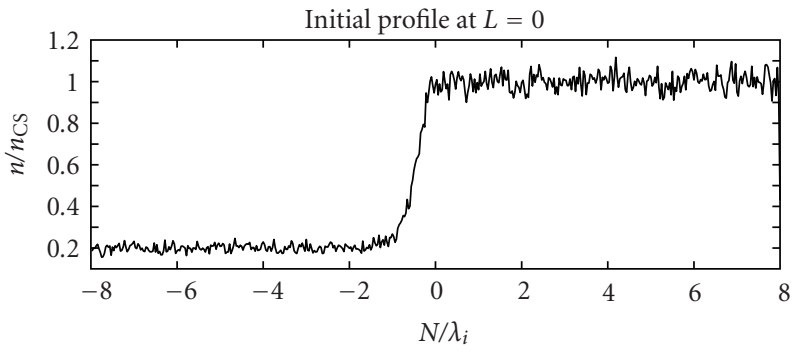

(b)

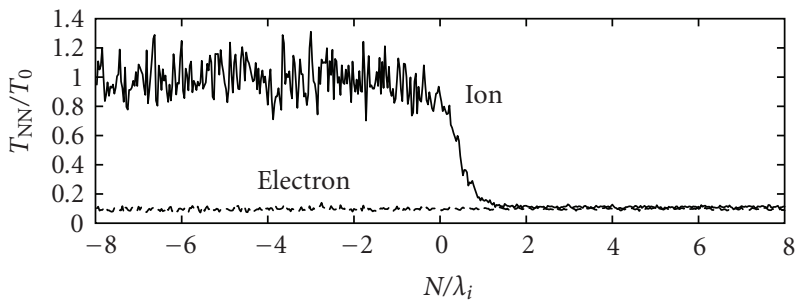

(d)

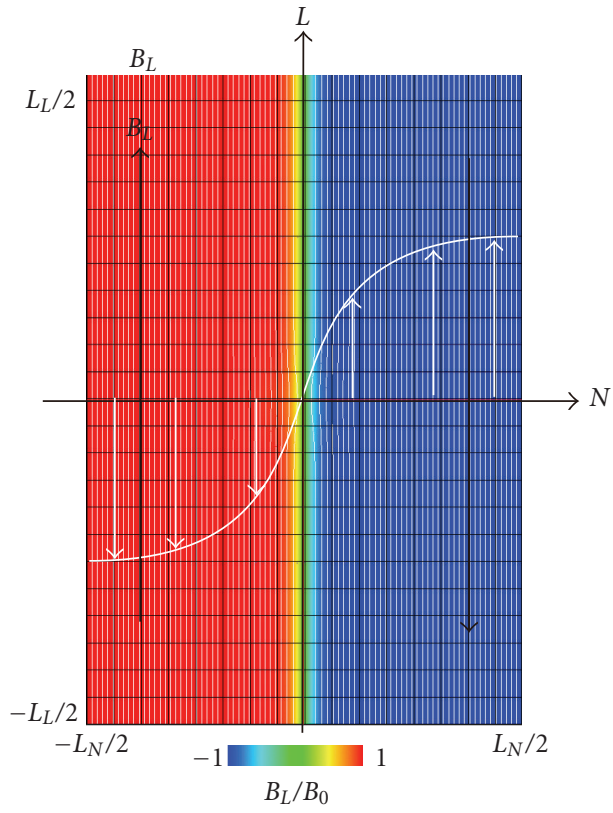

(f)

Figure 2: The initial configuration used in this study. From (a) to (d), the one-dimensional cuts of the $L$ component magnetic field $B_{L}$, the electron number density $n_{e}$, the ion and electron pressure tensor components $P_{i, \mathrm{NN}}$ (solid) and $P_{e, \mathrm{NN}}$ (dashed), and the ion and electron temperature tensor components $T_{i, \mathrm{NN}}$ (solid) and $T_{e, \mathrm{NN}}$ (dashed), respectively. (e) and (f) show the two-dimensional contours of the electron density and the $B_{L}$ component. The coordinate system is depicted in (e). White arrows in (f) depict how the shear flow is given in this study. Black arrows in (f) show the directions of the magnetic field.

The background temperatures are set to be $T_{i, \mathrm{BG} 1}=T_{e, \mathrm{BG} 1}=$ $T_{e, \mathrm{BG} 2}=T_{e, \mathrm{CS}}$. Then, $T_{i, \mathrm{BG} 2}$ (the ion temperature on the magnetospheric side) is determined to satisfy the pressure balance. After some easy calculation, the ion temperature is found to be $T_{i, \mathrm{BG} 2}=9 T_{e, \mathrm{CS}}$. The initial plasma beta in the magnetosheath is 0.22 . We note that this low beta magnetosheath is one of the limitations of our study.

Hereinafter, magnetic field and density will be normalized by $B_{o}$ and $n_{0}$, respectively. The ion inertial length $\lambda_{i}=c / \omega_{\mathrm{pi}}$ based on $n_{0}$ and the inverse ion gyrofrequency $\Omega_{i}^{-1}$ based on $B_{o}$ are the units for spatial and time scales, respectively, and velocities will be measured in the magnetosheath ion Alfvén speed unit $V_{A i}=B_{0} /\left(4 \pi m_{i} n_{0}\right)^{1 / 2}\left(m_{i}\right.$ : the ion mass). The electric field is normalized by $B_{o} *\left(V_{A i} / c\right)$, where $\mathrm{c}$ is the light speed. In this normalization, the magnetosheath electron inertial length is $\lambda_{e}=M^{-1 / 2}$ ( $M$ : the ion-to-electron mass ratio $\left.m_{i} / m_{e}\right)$. We set the electron plasma frequency to electron gyrofrequency ratio to be $\tau=\omega_{p e}=/ \Omega_{e}=1$ to 


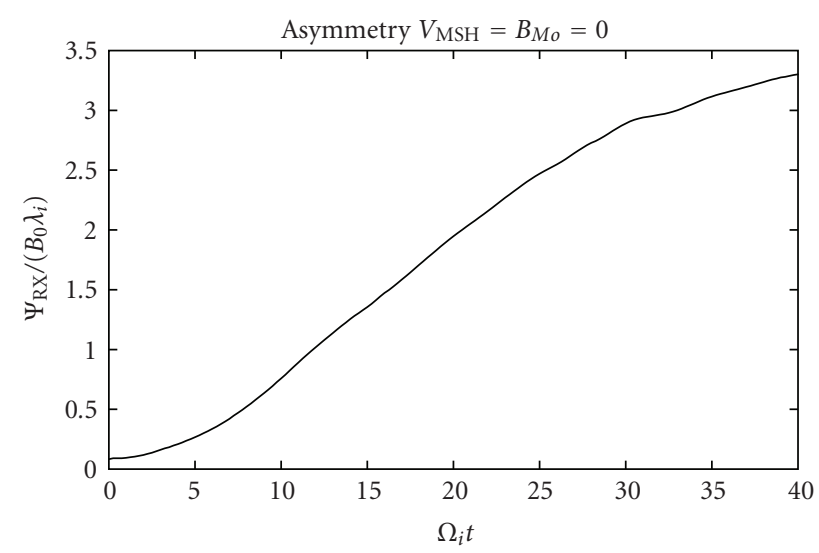

(a)

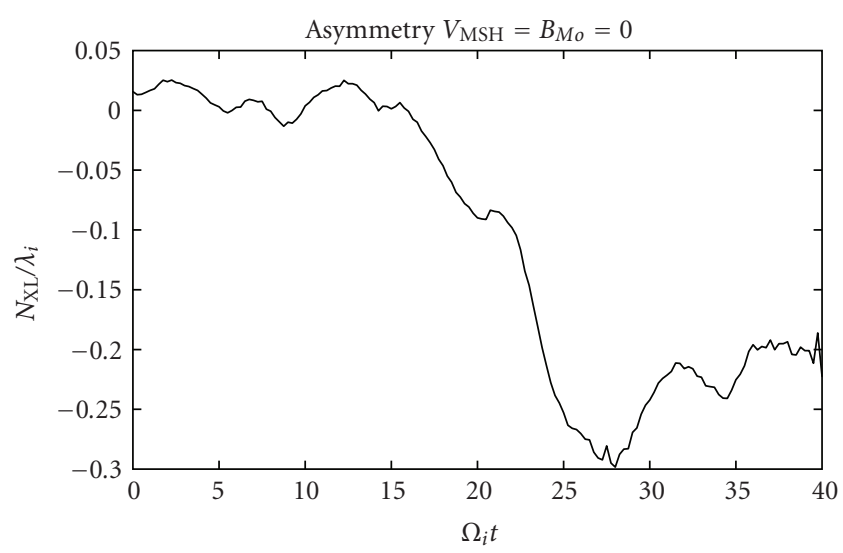

(b)

FIgURE 3: The result of $V_{\mathrm{MSH}}=B_{M o}=0$ case with density asymmetry. Temporal developments of (a) the reconnected flux and (b) $N$ coordinate of the X-line are shown. The X-line position shifts to the magnetospheric (negative $N$ ) side between $t=15$ and 30 .

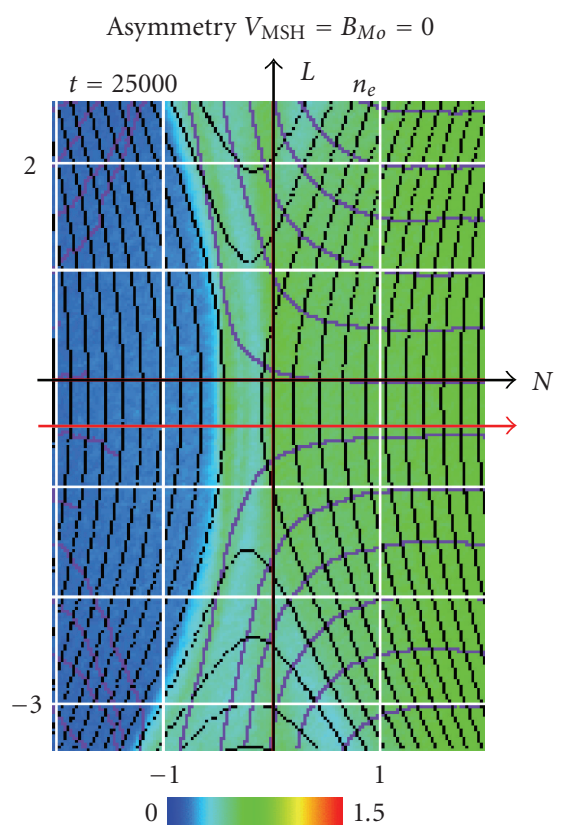

(a)

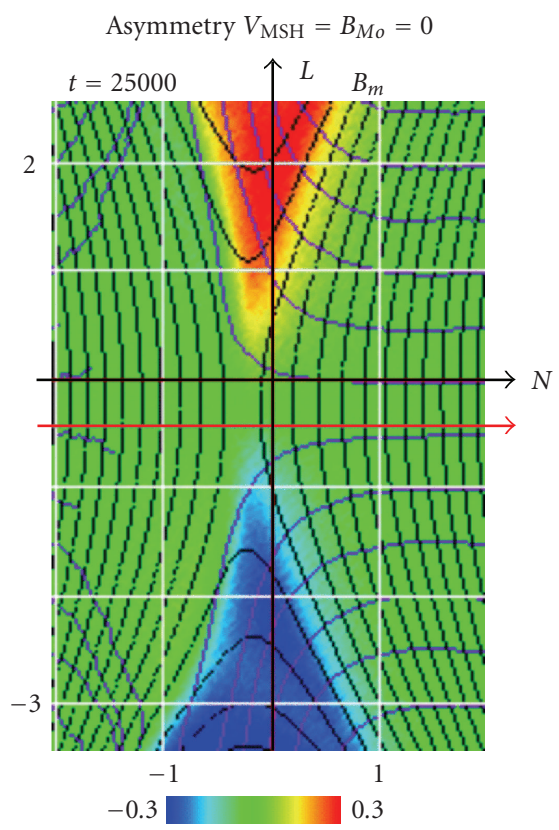

(b)

FIgURE 4: Zooming into the X-line region for the $V_{\mathrm{MSH}}=B_{M o}=0$ case. (a) The density and (b) out-of-plane magnetic field $B_{M}$ patterns in the area of $\left[L_{\mathrm{XL}}-3, L_{\mathrm{XL}}+3\right] \times\left[N_{\mathrm{XL}}-2, N_{\mathrm{XL}}+2\right]$ are shown. The time is $t=25$. The black and purple lines show the field lines and the ion streamlines, respectively.

reduce the computational cost, whereas the realistic value is $\sim 40$. In the present study, we do not expect $\tau$ to play a major role in the two-dimensional cases, as was recently suggested by Haijima et al. [31]. $M=100$ and thus $c / V_{A i}=$ 10 in our study.

Figure 2 shows the initial configuration of this study. Panels (a) (d) show the one-dimensional cuts along the $N$ axis of various parameters. From top to bottom, the magnetic fields $B_{L}$, the electron plasma density $n_{e}$, the ion and electron NN-pressure tensor elements $P_{i, \mathrm{NN}}$ (solid) and $P_{e, \mathrm{NN}}$ (dashed), and the ion and electron NN-temperature tensor elements $T_{i, \mathrm{NN}}$ (solid) and $T_{e, \mathrm{NN}}$ (dashed) are presented.
Two-dimensional structures of electron number density $n_{e}$ and the reconnecting magnetic field $B_{L}$ are shown in Panels (e) and (f). The LMN-coordinates are shown in Panel (e).

It is noted that the pressure balance initialization is different from that of Swisdak et al. [24]. While the initial pressure balance of Swisdak et al. [24] was under the varying magnetic field strength across the current layer and thus there was nonzero pressure gradient at the center of the current sheet, there is no pressure gradient at the center in our case.

In some of the cases studied in this paper, we will have a sheared flow and/or nonzero out-of-plane magnetic 


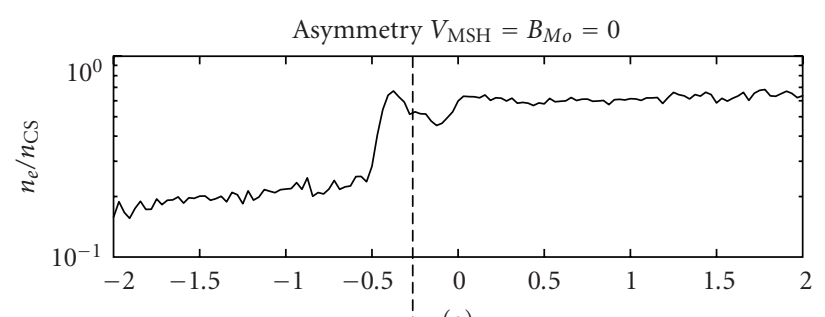

(a)

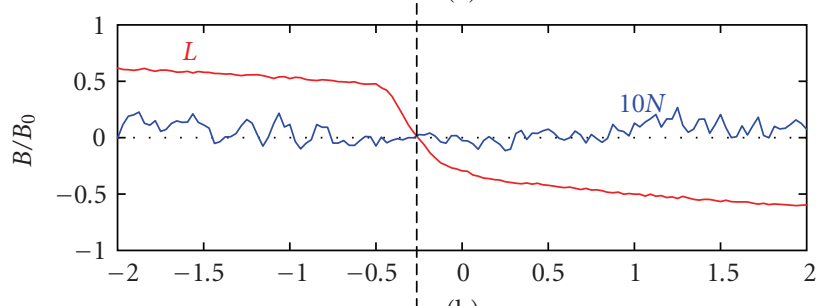

(b)

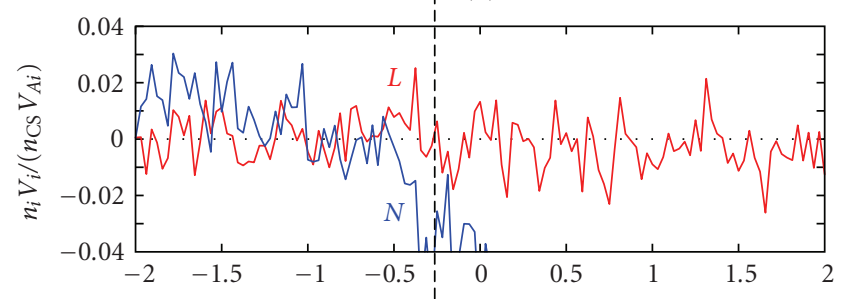

(c)

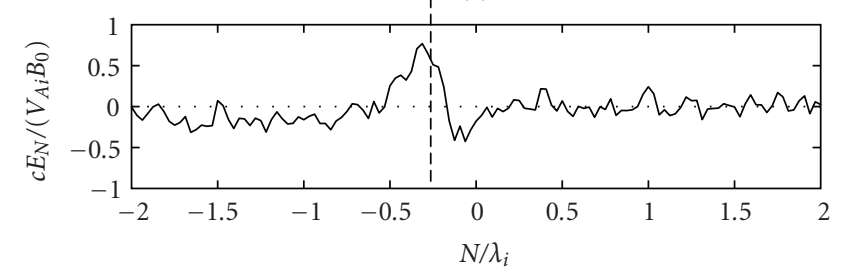

(d)

Figure 5: One-dimensional cuts of various parameters along the $N$ axis at the X-line position (along the red arrow shown in Figure 4). From top to bottom, (a) $n_{e}$, (b) $B_{L}$ (red) and $B_{N}$ (blue, multiplied by 10), (c) $n_{i} V_{\mathrm{iL}}$ (red) and $n_{i} V_{\mathrm{iN}}$ (blue), and (d) $E_{N}$, respectively. The dashed line at $\mathrm{X}_{N}=-0.25$ indicates the $\mathrm{X}$-line position (where $B_{L}=0$ in Panel (b)).

field. Sheared flow is set up by giving oppositely directed background bulk flow $\pm 0.5 V_{\mathrm{MSH}}$ in the simulation frame. A schematic of a sheared flow is given in Figure 2(f) by the white arrows. Here, $V_{\mathrm{MSH}}$ denotes the magnetosheath flow velocity measured in the magnetosphere-rest frame. With this choice of the simulation frame of reference, the system is symmetric in the absence of the density asymmetry. The guide field $B_{M o}$ is uniformly added in whole simulation domain (as mentioned already, this is different from the cases studied in Swisdak et al. [24]).

In addition to the Harris magnetic field, a Gaussiantype perturbation vector potential is introduced to set an $\mathrm{X}$-line at the center of the box. The amplitude of the initial perturbation in the component normal to the current sheet is $\delta B_{N}=0.05$.

Periodic boundary conditions are imposed in the $L$ direction while conducting walls are set at the $N$ boundaries. The simulation box is $\left[-L_{L} / 2: L_{L} / 2\right] \times\left[-L_{N} / 2: L_{N} / 2\right]$ in the normalized unit, with $L_{L}=24$ and $L_{N}=16$. The boundary conditions do not have any effects on the results shown in this paper. The half-thickness of the current layer $D$ is set to be 0.5 . The spatial grid of $\Delta x=0.9 \lambda_{\text {De }}\left(\lambda_{\text {De }}\right.$ : Debye length) and the time step of $\Delta t=0.15625 \Omega_{e}^{-1}\left(\Omega_{e}\right.$ : the electron gyrofrequency) are adopted, respectively. 320 particles per grid represent the unit density.

\section{Key Quantities to Be Inspected in This Study}

The location of the X-line is denoted by $\left(L_{\mathrm{XL}}, N_{\mathrm{XL}}\right)$. In the same way, the velocities of the $\mathrm{X}$-line, ion velocity at the $\mathrm{X}$-line, and the electron velocity at the $\mathrm{X}$-line are denoted by $\left(V_{L, \mathrm{XL}}, V_{N, \mathrm{XL}}\right),\left(V_{\mathrm{iL}, \mathrm{XL}}, V_{\mathrm{iN}, \mathrm{XL}}\right)$, and $\left(V_{\mathrm{eL}, \mathrm{XL}}\right.$, $\left.V_{\text {eN,XL }}\right)$, respectively. Note that these velocities are measured in the simulation reference frame, and translation to the magnetosphere-rest frame requires correction by $0.5 V_{\mathrm{MSH}}$ in the $L$-component. The reconnected flux will be often used to present the reconnection activity and is defined by $\Psi_{\mathrm{RX}}=$ $0.5 \int\left|B_{N}(L, 0)\right| d L$.

\section{Simulation Results}

In the presence of density asymmetry, it is now known that the ion stagnation point is shifted to the magnetospheric (low density) side of the X-line $[14,15]$. For a clarification purpose, this simplest case with neither shear flow nor guide field is introduced briefly at the beginning. Then more effects will be added in the subsections that follow.

4.1. The $V_{M S H}=B_{M o}=0$ Case. Figure 3 shows the results of the $V_{\mathrm{MSH}}=B_{M o}=0$ case. Panel (a) shows the time history of reconnected flux $\Psi$. Panel (b) shows the time history of $N_{\mathrm{XL}}$. The X-line position starts to shift toward the magnetospheric side $(N<0)$ at $t \sim 16$. Then until $t \sim 30$, the X-line continues to move at a roughly constant speed of $V_{N, \mathrm{XL}} \sim 0.023$. This result obtained by our particle code is consistent with the previous studies using fluid models $[14,15]$.

Figure 4 shows the two-dimensional contour of the (a) ion density and (b) out-of-plane magnetic field $B_{M}$ at $t=25$. At this time, magnetic reconnection is still going on (Figure 3(a)). Only a small fraction around the X-line $[L, N]=[-3.5: 2.5,-2: 2]$ is shown. The black and purple lines represent the field lines and the ion number density flux streamlines $n_{i} V_{i}$, respectively. The streamlines clearly show the asymmetric inflow toward the X-line from the magnetosheath side. $B_{M}$ is positive at $L>0$ and vice versa. This dipolar feature of the Hall field is the characteristics of a density asymmetry case (e.g., $[25,27])$ and has been seen in observations [32]. The red horizontal line is the path along which the $N$ directional profiles of various parameters are sampled (see Figure 5).

The asymmetric inflow is shown in a more explicit manner. Shown in Figure 5 are the $N$-directional profiles of various parameters at $L=L_{\mathrm{XL}}$. From top to bottom, (a) $n_{e}$, (b) $B_{L}$ (red) and $B_{N}$ (blue, multiplied by 10$)$, (c) $n_{i} V_{\mathrm{iL}}$ (red) and $n_{i} V_{\text {iN }}$ (blue), and (d) $E_{N}$ are shown. The black vertical 


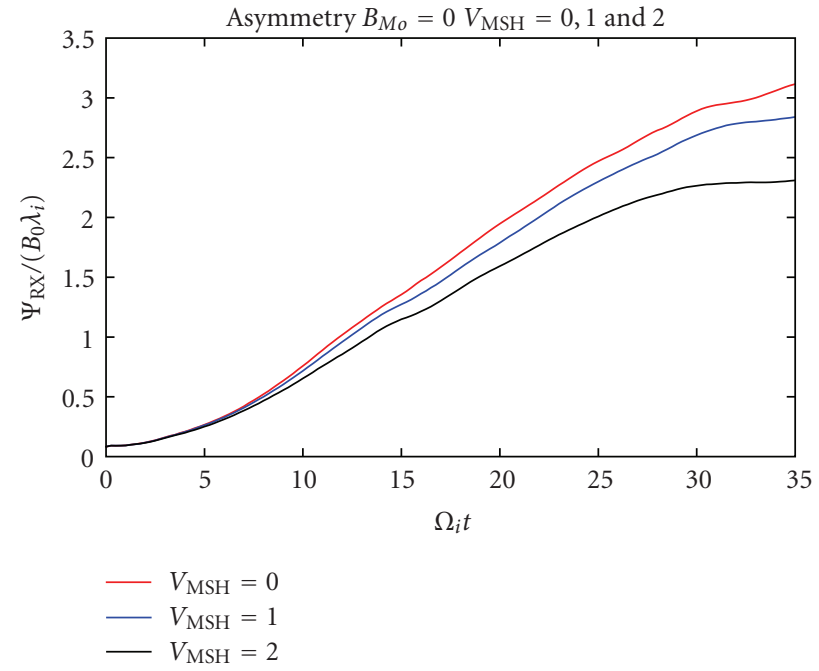

(a)

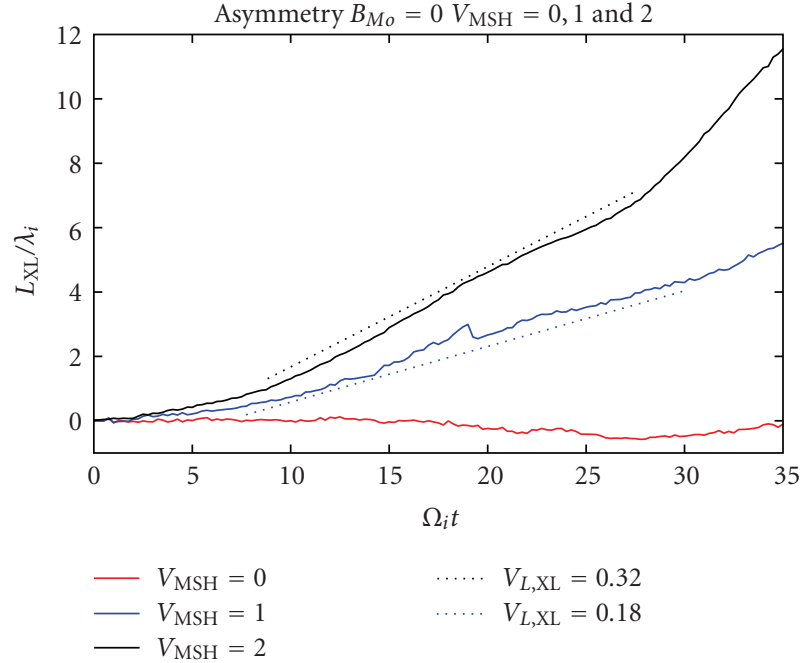

(b)

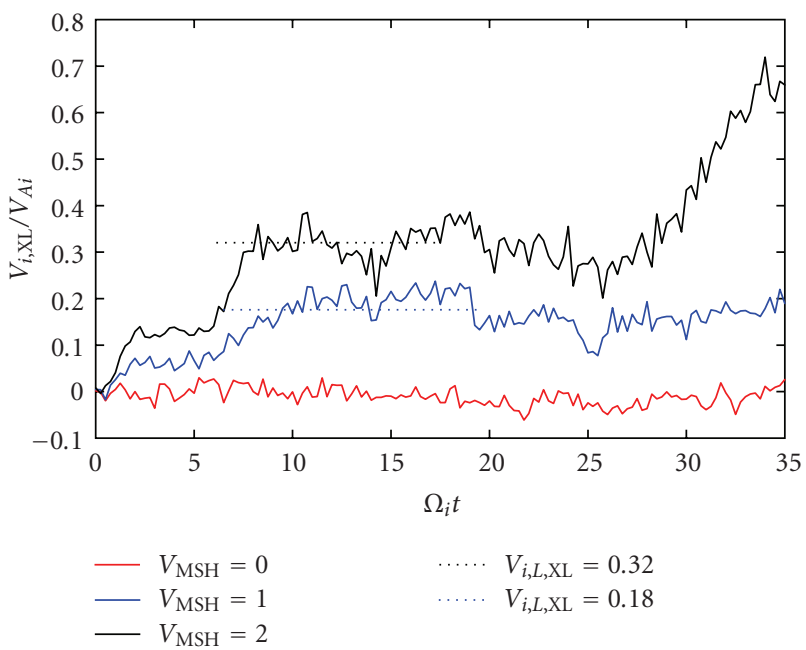

(c)

FIgURE 6: The results of nonzero $V_{\text {MSH }}$ cases without guide field. (a) Growth curves of reconnected flux. (b) The $L$-coordinates of the X-lines versus time. (c) The X-line speeds compared with the ion and electron flow at the X-line.

line depicts the location of the X-line $N_{\mathrm{XL}}$ (determined in Panel b). In Panel (c), while the ion stagnation point is located between $-1<N<-0.5$, it is clear that the ion stagnation point is more to the magnetospheric side than the $\mathrm{X}$-line.

4.2. Combination of Density Asymmetry and Sheared Flow. Section 4.1 showed that the magnetosheath ions flow across the X-line into the magnetospheric side. On the other hand, we are aware that there is a flow component along the magnetopause (the $L$ direction) in the magnetosheath side away from the equator. Then, it is natural to expect that the $L$ component of the magnetosheath ion flow at the X-line site is not zero. In this section, we study how such a slanted ion inflow affects the properties of the $X$-line. Here, guide field is set to be zero. How the shear flow is set (i.e., the frame of reference) is described in Figure 2(f).
Figure 6 compares the results from various $V_{\mathrm{MSH}}$ (the velocity jump across the magnetopause) cases. Panel (a) shows the time histories of $\Psi_{\mathrm{RX}}$. The red, blue, and black lines represent the $V_{\mathrm{MSH}}=0,1$, and 2 cases, respectively. One can see the reconnection activity reduces with increasing $V_{\mathrm{MSH}}$, but the effects are not very dramatic up to $V_{\mathrm{MSH}}=2$. Panel (b) shows the position of the X-line $\left(L_{\mathrm{XL}}\right)$ versus time. The dotted lines depict the slopes and thus the speeds of the $\mathrm{X}$ line motion $V_{L, \mathrm{XL}}$ during the reconnection stage. $V_{L, \mathrm{XL}}$ are 0.18 and 0.32 for the $V_{\mathrm{MSH}}=1$ and $=2$ cases, respectively. Comparison between Panels (a) and (b) suggests that the Xline sliding is an ongoing process of magnetic reconnection. Seen in the magnetospheric-rest frame, the speed of the moving $\mathrm{X}$-lines is $\sim 0.7$ times of the magnetosheath flow speed in both cases $\left(V_{L, \mathrm{XL}}=0.68\right.$ in the $V_{\mathrm{MSH}}=1$ case and $=1.32$ in the $V_{\mathrm{MSH}}=2$ case, resp.). Panel (c) shows the $L$ component of the ion flow at the X-line $V_{\mathrm{iL}, \mathrm{XL}}$, which is nonzero. The dotted black and blue horizontal lines 


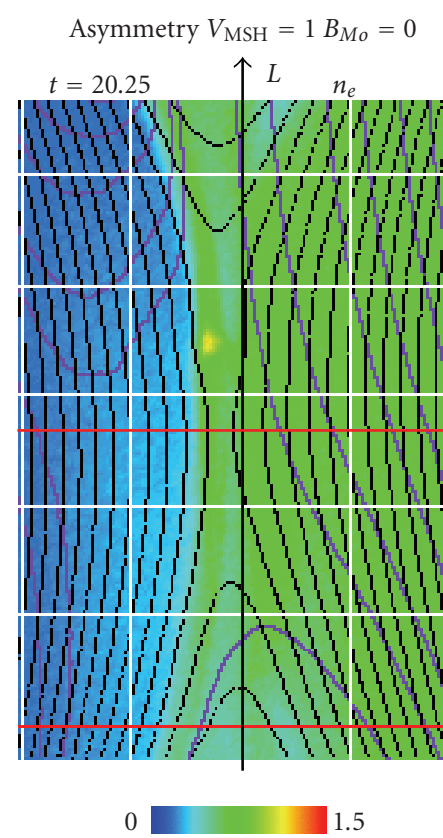

(a)

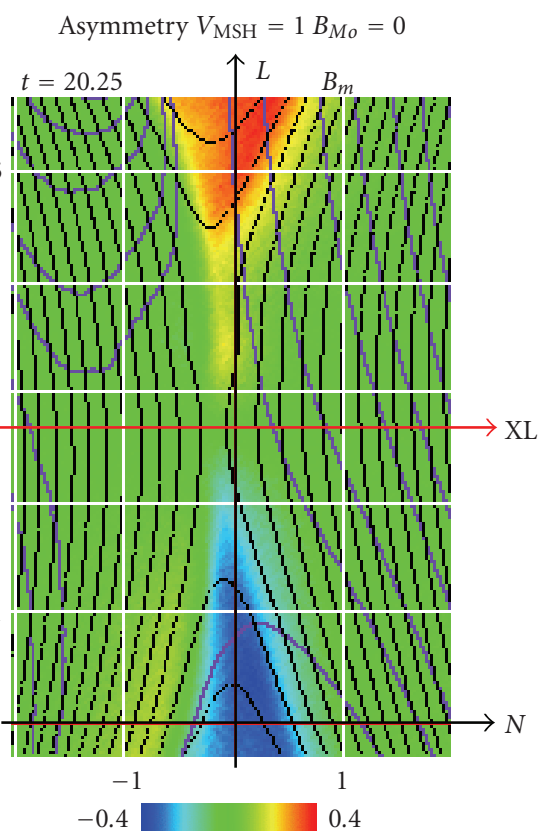

(b)

FIgure 7: Zooming into the X-line region for the $V_{\mathrm{MSH}}=1$ and $B_{M o}=0$ case. (a) Density and (b) $B_{M}$. The black and purple curves are the field lines and the ion streamlines, respectively. The spatial dimension is $\left[L_{\mathrm{XL}}-3, L_{\mathrm{XL}}+3\right] \times\left[N_{\mathrm{XL}}-2, N_{\mathrm{XL}}+2\right]$. The time is $t=20.25$.

depict $V_{L, X L}$ as references. One can see that there is nice agreement between $V_{\mathrm{iL}, \mathrm{XL}}$ and $V_{L, \mathrm{XL}}$. The slanted inflow of the magnetosheath ions makes nonzero $V_{\mathrm{iL}, \mathrm{XL}}$ at the $\mathrm{X}$-line and induces the $\mathrm{X}$-line to move in the same direction at the same speed, $V_{L, \mathrm{XL}} \sim V_{\mathrm{iL}, \mathrm{XL}}$.

Figure 7 is the same format as Figure 4 except for the $V_{\mathrm{MSH}}=1$ case at $t=20.25$. The area covered by each panel is $[-0.3: 5.7] \times[-2: 2]$. Comparison between Figures 4(b) and 7(b) presents that $B_{M}$ structure has less significant difference in the parameter range considered here. The red vertical arrow is the path along which the one-dimensional cuts at $L=L_{\mathrm{XL}}$ shown in Figure 8 are made.

Figure 8 shows the $N$-directional cuts at the X-line position $L=L_{\mathrm{XL}}$. The format is the same as Figure 5 . In Panel (b), the X-line has shifted into the magnetospheric side at $N_{\mathrm{XL}}=-0.1$ (vertical black dashed line) while in Panel (c), the ion stagnation point is around $N=-0.8 . n_{i} V_{\mathrm{iN}}$ shown in Panel (c) indicates that the magnetosheath ions flow across the X-line toward the magnetospheric side and $n_{i} V_{\mathrm{iL}}$ at the X-line has a nonzero positive value.

4.3. Combination of Density Asymmetry and out-of-Plane Magnetic Field. Section 4.2 has shown that a slanted ion inflow to the X-line forces it to slide along the $L$-direction. The slanted inflow was realized by the combination of density asymmetry and shear flow. One may also expect that the combination of density asymmetry and nonzero guide field $B_{M}$ is to produce a slanted inflow of ions (See Figure 1). On the other hand, when nonzero guide field is present, the inplane current pattern is formed in the course of reconnection and there generated nonzero electron flow at the $\mathrm{X}$-line, which may also drive the $\mathrm{X}$-line to move. The case discussed in this subsection has the uniform guide field of $B_{M o}=-0.5$ and is without a shear flow $V_{\mathrm{MSH}}=0$.

Figure 9 shows the results. Panels (a) and (b) show the time histories of $\Psi_{\mathrm{RX}}$ and $L_{\mathrm{XL}}$, respectively. In Panel (b), time-space evolution of the $B_{N}$ component along the reversing- $B_{L}$ curve (i.e., $B_{L}=0$ ) is shown. For the convenience, the $B_{L}=0$ curve is projected onto the $L$-axis. The black stripe elongated to the upright direction shows the time evolution of the region where $B_{N}=0$ and thus shows the X-line motion. The X-line starts to slide at $t \sim 10$ with the constant velocity of $V_{L, \mathrm{XL}} \sim 0.15$ until $t \sim 30$. Panel (c) compares this $V_{L, \mathrm{XL}}$ (horizontal dashed line) with the ion and electron bulk flow velocities at the X-line, $V_{\mathrm{iL}, \mathrm{XL}}$ (dotted) and $V_{\mathrm{eL}, \mathrm{XL}}$ (dotted). All the three components are in the same direction (positive- $L$ ). The large difference in the velocity component between the ions and the electrons indicates that there is significant current density along the $L$-axis at the Xline, which is not present in the initial condition. The X-line speed $V_{L, \mathrm{XL}}=0.15$ is larger than the rather small ion velocity of $V_{\mathrm{iL}, \mathrm{XL}}=0.05$. On the other hand, the electrons have a large flow of $V_{\mathrm{eL}, \mathrm{XL}}=1$. While a definitive conclusion cannot be drawn only from these results, one would tend to think that the small ion flow is not sufficient to drive the faster $\mathrm{X}$-line motion and that the role of the electrons cannot be neglected.

Figure 10 shows the two-dimensional contours of (a) density and (b) $B_{M}$ at $t=16$. The format is the same as Figure 7. At the $\mathrm{X}$-line, one can see that there is $\partial B_{M} / \partial N>0$. The cut along the $N$-axis at the $\mathrm{X}$ line shown in Figure 11 tells this more explicitly. Panels 

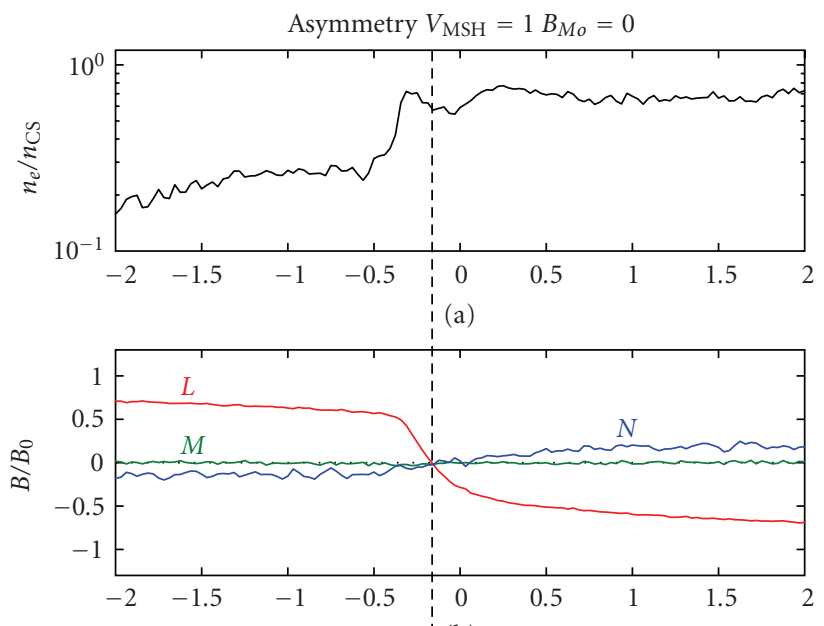

i (b)

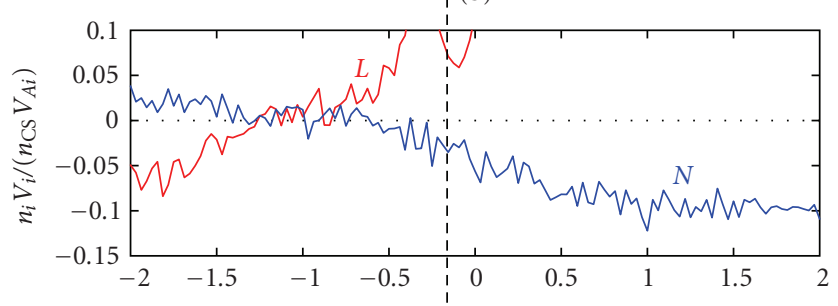

(c)

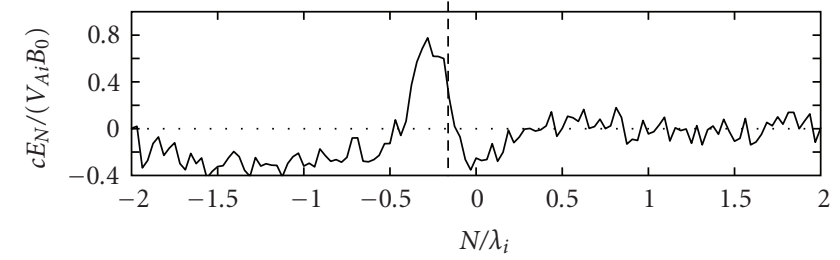

(d)

FIGURE 8: Various parameters along the red arrow shown in Figure 7. In the same format as Figure 5, Panel (c) clearly indicates that the ion flow component at the X-line $V_{\mathrm{iL}, \mathrm{XL}}$ is nonzero and positive.

(a) (d) show the magnetic field, the ion flow, the electron flow, and $\partial B_{M} / \partial N$, respectively. The X-line, which, unlike the zero-guide field cases shown previously, somehow has shifted to the magnetosheath side, is filled with the magnetosheath ions having the small positive $L$-component velocity. At the same time, the negative $L$-component of the current density implied by $\partial B_{M} / \partial N>0$ at the Xline is sustained by the fast electron flow in the positive- $L$ direction.

Coming back to Figure 10, in the quadrant above the $\mathrm{X}$-line and on the magnetosheath side, both the density and the $B_{M}$ component show peculiar features that appear through the combination of the density asymmetry and the nonzero guide field. Here, the density in the upward directed jet is larger than the magnetosheath density $(=1)$. This signature is consistent with an asymmetric reconnection with a guide field work by Pritchett [25]. Both the density and $B_{M}$ perturbation patterns located near the separatrices are similar to what is understood in guide field magnetic
TABLE 1: Summary of the results from the simpler cases. $B_{M o}$ : guide field, $V_{\mathrm{MSH}}$ : velocity jump across the magnetopause, $V_{\mathrm{XL}, \text { sim }}$ : the $X$ line speed measured in the simulation frame.

\begin{tabular}{lcc}
\hline$B_{M o}$ & $V_{\mathrm{MSH}}$ & $V_{\mathrm{XL}, \mathrm{sim}}$ \\
\hline-0.5 & 0 & +0.15 \\
+0.5 & 0 & -0.15 \\
0 & 1 & +0.18 \\
\hline
\end{tabular}

reconnection with a symmetric density situation although these patterns are skewed somewhat due to the inflow asymmetry. Since the density on the magnetospheric side is 0.2 , a natural expectation is that the jet density would be between 0.2 and 1. In addition, there is a band of weak $B_{M}$ located at the outer part of the jet (the reddish part with $B_{M} \sim-0.2$ ). This is also counter to a natural expectation that the guide field component is compressed $(<-0.5)$ in the jet. Figure 12 shows the one-dimensional cut along the upper red arrow depicted in Figure 10. The format is the same as Figure 5, except that Panels (c) and (d) are now for the ion and electron flows, respectively. Panel (a) shows that the density at $N=0.5$ increases up to $n_{e}=1.5$. In this location, the ion jet $V_{\mathrm{iL}}$ is nonnegligibly large. The high-density part of the jet is situated between the magnetosheath and the jet showing $E_{N}>0$ (pointing outward to the magnetosheath side, Panel (e)). The ions coming into the jet from the magnetosheath side, when unmagnetized, would sense this $E_{N}>0$ as the repelling force to push the ions back toward the magnetosheath side. Then the ions are confined in the magnetosheath side of the jet. This would explain the origin of the high-density part. In the second and fourth (first and third) quadrants of Figures $10(\mathrm{a})$ and 10(b), the density decreases (increases) and $B_{M}$ is compressed (rarefied). Previous guide field reconnection works with symmetric density also discussed these structures and suggested that this structure arises due to the physics of the kinetic Alfvén wave in association with intense electric fields [18, 33-35].

The X-line motion induced by the combination of density asymmetry and the uniform guide field is not necessary trivial, and we have double-checked the results by performing a run with a smaller $M=25$ but with a larger simulation box $L_{L}=192$ (with $D=1$ ). Figure 13 shows the X-line motion in the same format as Figure 9(b). From the slope, one obtains $V_{L, \mathrm{XL}} \sim 0.15$, which is in excellent agreement with the $M=100$ case. This run also enables us to confirm the constancy of the X-line speed for an extended time period in a larger box. Figure 14 shows twodimensional contours of (a) number density and (b) out-ofplane magnetic field. One can clearly see that the peculiar features in the upward jet, namely, the higher density in the magnetosheath side half and the weak $B_{M}$ attached at the edge on the magnetosheath side, elongate away from the $\mathrm{X}$ line implying that they are not the features local to the X-line region.

4.4. Combination of Density Asymmetry, Shear Flow, and outof-Plane Magnetic Field. We have learned that combination 


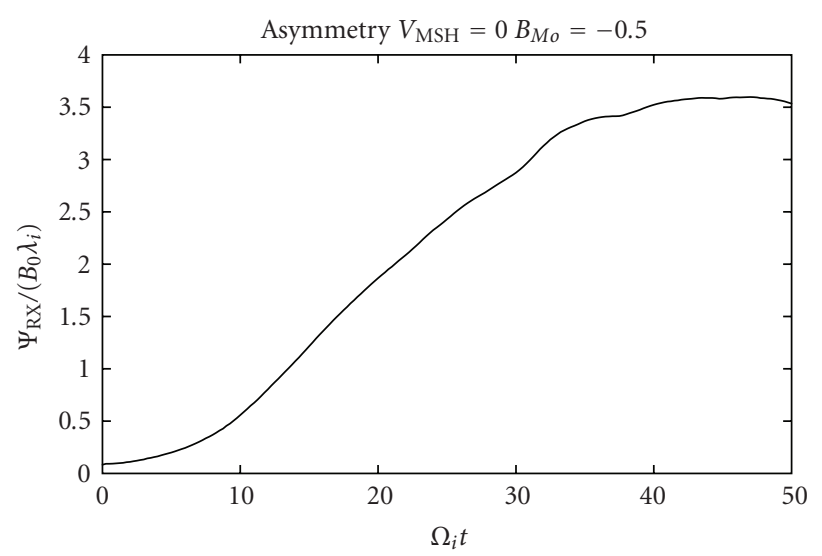

(a)

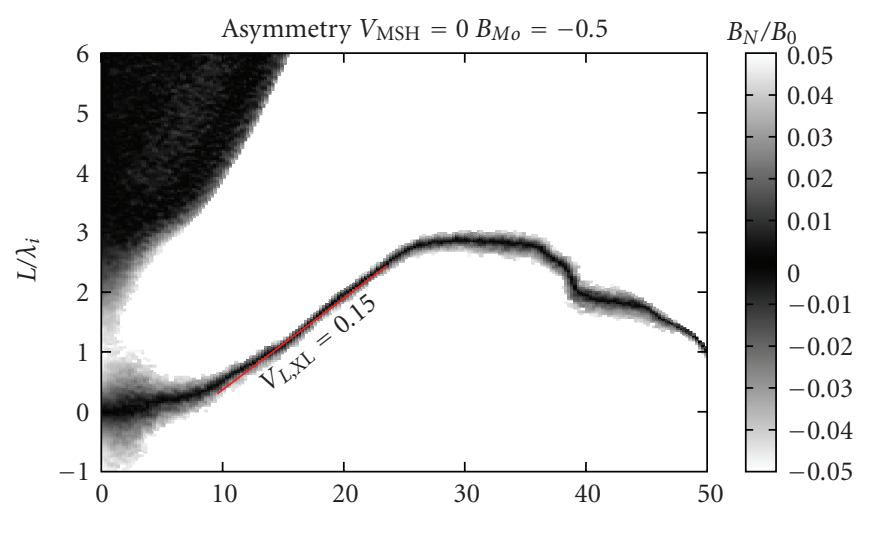

(b)

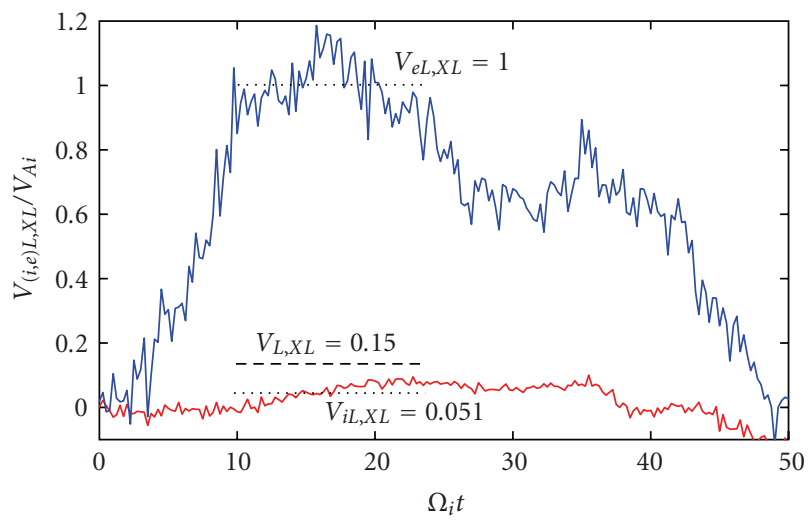

(c)

Figure 9: Results of the nonzero guide field $B_{M o}=-0.5$ case without velocity shear $\left(V_{\mathrm{MSH}}=0\right)$. (a) Growth of reconnection flux, (b) grayscale contour of $B_{N}$ along the $B_{L}=0$ curve in the $L$-time diagram depicting the X-line motion, and (c) the X-line motion speed compared with ion and electron flow speed at the $\mathrm{X}$-line.

of density asymmetry and shear flow (in Section 4.2), or of density asymmetry and nonzero guide field (in Section 4.3), makes the $\mathrm{X}$-line to slide along the $L$ direction. While there may well be various other ways to create an Xline motion, the two items combined with the density asymmetry considered here are the ones well expected at the magnetopause. In other words, the X-lines driving the magnetopause reconnection are most likely to be in motion. The direction of the X-line motion is determined by (1) the flow direction in the magnetosheath for the shear flow only case and (2) the sign of the out-ofplane magnetic field for the nonzero guide field only case. Then, an interesting question is how an X-line behaves when both the shear flow and guide-field are simultaneously present, and especially when they counteract to each other in determining the direction of the X-line motion.

Table 1 is the summary of the results described so far that have relevance to the run studied in the next subsection. $V_{\mathrm{XL}, \text { sim }}$ is the $\mathrm{X}$-line speed in the simulation frame. $B_{\mathrm{Mo}}= \pm 0.5$ alone or $V_{\mathrm{MSH}}=1$ alone induces almost the same X-line sliding speed. The direction, however, can be opposite according to the sign of $B_{M o}$ (as mentioned, we think that the frame in which these velocities are measured is suited for this kind of discussion). The counter-acting case would be the most interesting one to study and the results are shown below.

4.4.1. The $B_{M o}=0.5$ and $V_{M S H}=1$ Case. Figure 15 shows the results of the $\left(V_{\mathrm{MSH}}, B_{M o}\right)=(1,0.5)$ case. Previous results show that the effects of $V_{\mathrm{MSH}}=1$ would push the X-line in the positive $L$-direction while those of $B_{M o}=0.5$ in the negative $L$-direction. One can see in Panel (a) that the X-line starts to slide from $t=10$ in the positive $L$-direction with $V_{L, \mathrm{XL}}=0.14$. This $\mathrm{X}$-line speed is almost the same as the $V_{\mathrm{MSH}}=1$ alone case (Table 1$)$. Panel $(\mathrm{b})$ shows $V_{\mathrm{iL}, \mathrm{XL}}=$ 0.35 and $V_{\mathrm{eL}, \mathrm{XL}}=-0.5$. The $\mathrm{X}$-line motion is in the same direction as the ion flow and is opposite to the electron flow.

What is interesting here is the fact that $V_{\mathrm{iL}, \mathrm{XL}}$ is faster than the $V_{\mathrm{MSH}}=1$ alone case (0.18). The argument in Figure 1 implied that the guide field $B_{M o}>0$ would make the ion flow to be slower, but this turns out not to be the case. What is more interesting, despite this accelerated ion flow, the Xline speed is slightly slower. This indicates that there should be something else other than the ion flow to determine the 


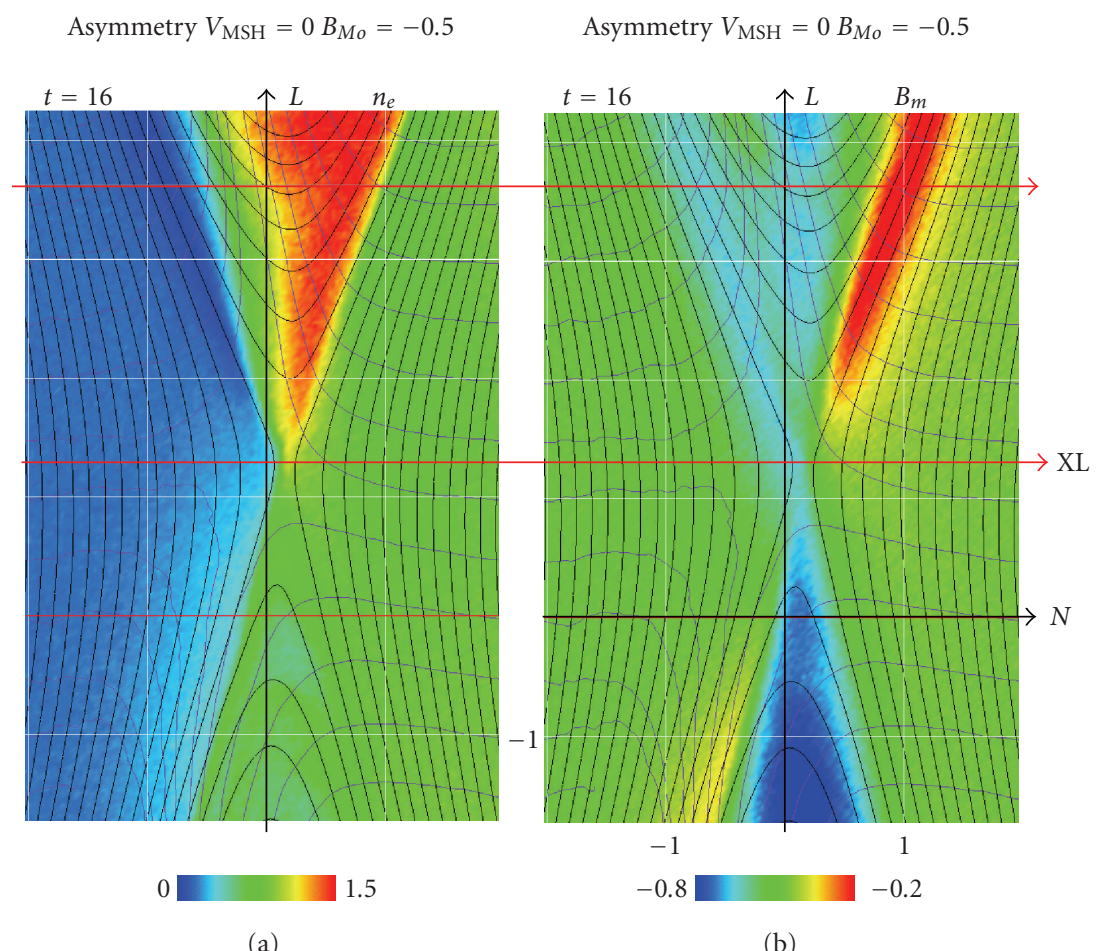

Figure 10: Results from the $\left(V_{\mathrm{MSH}}, B_{M o}\right)=(0,-0.5)$ case shown in the same format as Figure 7 . The spatial dimension is $\left[L_{\mathrm{XL}}-3, L_{\mathrm{XL}}+\right.$ $3] \times\left[N_{\mathrm{XL}}-2, N_{\mathrm{XL}}+2\right]$. The time is $t=16$. The part of the upward jet having density higher than the magnetosheath value is accompanied by the band of $B_{M} \sim 0$ on the magnetosheath side of it.

TABLE 2: Summary of all the cases studied in this paper. $B_{M o}$ : guide field, $V_{\mathrm{MSH}}$ : velocity jump across the magnetopause, $V_{\mathrm{XL}, \text { sim }}$ : the $X$-line speed measured in the simulation frame, $V_{\mathrm{iL}, \mathrm{XL}}$ : the $L$-component of the ion flow at the $X$-line, $V_{\mathrm{eL}, \mathrm{XL}}$ : the $L$-component of the electron flow at the $X$-line, and $V_{\mathrm{XL}, \mathrm{MSP}}$ : the $\mathrm{X}$-line speed in the magnetosphere-rest frame.

\begin{tabular}{|c|c|c|c|c|c|c|}
\hline$B_{M o}$ & $V_{\mathrm{MSH}}$ & $V_{\mathrm{XL}, \text { sim }}$ & $V_{\mathrm{iL}, \mathrm{XL}}$ & $V_{\mathrm{eL}, \mathrm{XL}}$ & $V_{\mathrm{XL}, \mathrm{MSP}}$ & $V_{\mathrm{XL}, \mathrm{MSP}} / V_{\mathrm{MSH}}$ \\
\hline-0.5 & 0 & 0.15 & 0.051 & 1 & 0.15 & N/A \\
\hline-0.5 & 0.5 & 0.28 & 0.26 & 1.21 & 0.53 & 1.06 \\
\hline-0.5 & 1 & 0.37 & 0.42 & 1.3 & 0.87 & 0.87 \\
\hline 0 & 1 & 0.18 & 0.18 & 0.4 & 0.68 & 0.68 \\
\hline 0 & 2 & 0.32 & 0.31 & 0.52 & 1.32 & 0.66 \\
\hline 0.5 & 0 & -0.15 & -0.051 & -1 & -0.15 & N/A \\
\hline 0.5 & 0.2 & -0.088 & 0.047 & -0.97 & 0.012 & 0.06 \\
\hline 0.5 & 0.4 & -0.027 & 0.1 & -0.84 & 0.17 & 0.43 \\
\hline 0.5 & 0.5 & 0.0047 & 0.14 & -0.78 & 0.25 & 0.51 \\
\hline 0.5 & 0.7 & 0.072 & 0.22 & -0.69 & 0.42 & 0.6 \\
\hline 0.5 & 1 & 0.14 & 0.35 & -0.5 & 0.64 & 0.64 \\
\hline
\end{tabular}

speed of the X-line motion. Indeed, the gradient of the guide field component at the X-line drives the fast electron flow in the opposite direction $(-0.5)$ and this may be the slowing down agent. That is, both ion and electron flow at the X-line seem to play roles in determining the direction and speed of the $\mathrm{X}$-line motion.

4.4.2. $V_{L, X L}$ from Various $\left(V_{M S H}, B_{M o}\right)$ Cases. When the sign of $B_{M o}$ is flipped $\left(\left(V_{\mathrm{MSH}}, B_{M o}\right)=(1,-0.5)\right.$ case $)$, the combined effects are found to speed up the X-line motion to $V_{L, \mathrm{XL}}=0.37$. The flow velocities at the $\mathrm{X}$-line are
$V_{\mathrm{iL}, \mathrm{XL}}=0.42$ and $V_{\mathrm{eL}, \mathrm{XL}}=1.3$, respectively. The flipping of the $B_{M}$ component leads to a substantial change in $V_{L, X L}$ (from 0.14 to 0.37 ) with only slight change in $V_{\mathrm{iL}, \mathrm{XL}}$ (from 0.35 to 0.42 ). In contrast, there is a substantial change in $V_{\text {eL,XL }}$ (from -0.5 to 1.3 ). These again suggest possible roles played by the electrons in regulating the X-line motion. Here, we try to learn more by studying the results from various sets of $\left(V_{\mathrm{MSH}}, B_{M o}\right)$ in this subsection. Table 2 shows all the cases studied.

Figure 16 shows the time history of reconnected flux for the four cases $\left(V_{\mathrm{MSH}}, B_{M o}\right)=(0,-0.5)$ (black), $(1,0)$ 


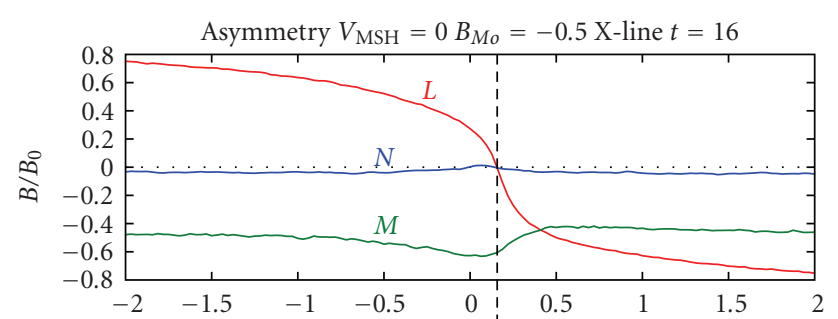

(a)

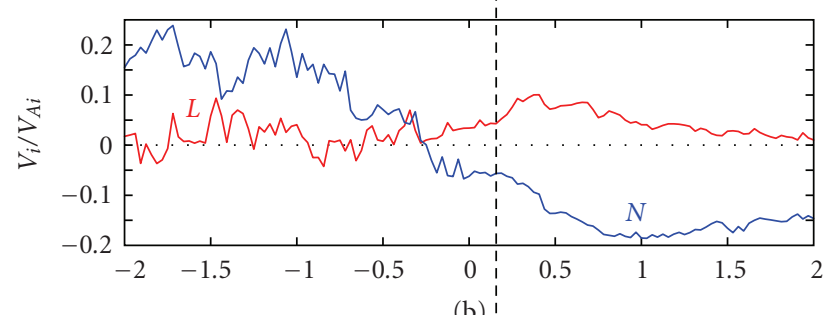

(b)

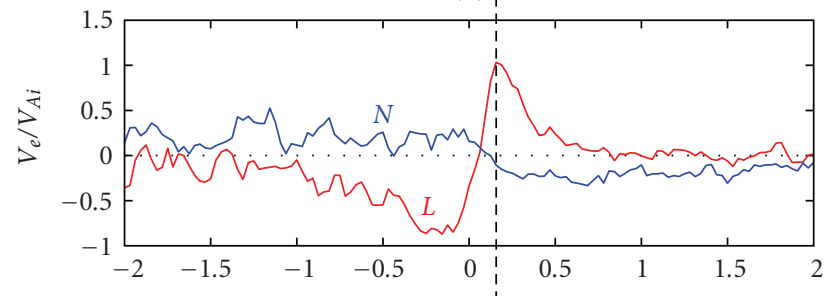

(c)

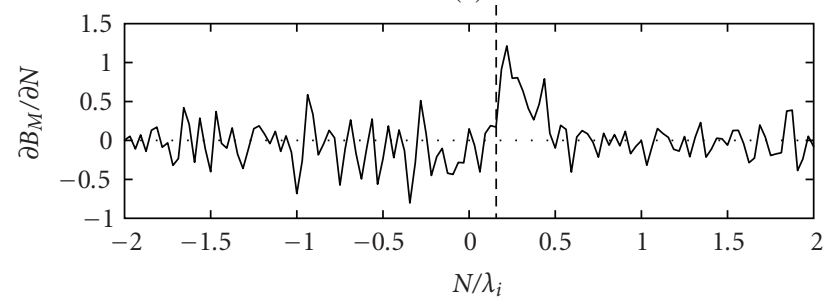

(d)

Figure 11: The cuts along the $N$-axis at the X-line of (a) $B$, (b) $V_{i}$, (d) $V_{e}$, and (d) $\partial B_{M} / \partial N$. The X-line is filled with the magnetosheath ions having the small positive $L$-component velocity. At the same time, the negative $L$-component of the current density implied by $\partial B_{M} / \partial N>0$ at the $\mathrm{X}$-line is sustained by the fast electron flow in the positive- $L$ direction.

(blue), $(1,0.5)$ (greed), and $(1,-0.5)$ (red), respectively. This figure is to confirm that there is no substantial difference in terms of reconnection history due to the shear flow, nonzero guide field, and their combinations within the range considered here.

Figure 17 (a) plots $V_{L, \mathrm{XL}}$ versus $V_{\mathrm{MSH}}$, with different $B_{M o}$ cases shown by different colors (blue, black, and red for $B_{M o}=-0.5,0$, and 0.5 , resp.). The general trends are (1) $V_{L, \mathrm{XL}}$ is larger for larger $V_{\mathrm{MSH}}$ and (2) $B_{M o}=-0.5$ (blue) cases give the fastest and $B_{M o}=0.5$ (red) cases give the lowest $V_{L, \mathrm{XL}}$ when $V_{\mathrm{MSH}}$ is the same. The most interesting feature is that the $\mathrm{X}$-line motion can be opposite to the magnetosheath flow when $V_{\mathrm{MSH}}$ is small and $B_{\mathrm{Mo}}=0.5$ (red). These clearly indicate that it is not only the ion flow that determines the direction of the X-line motion.
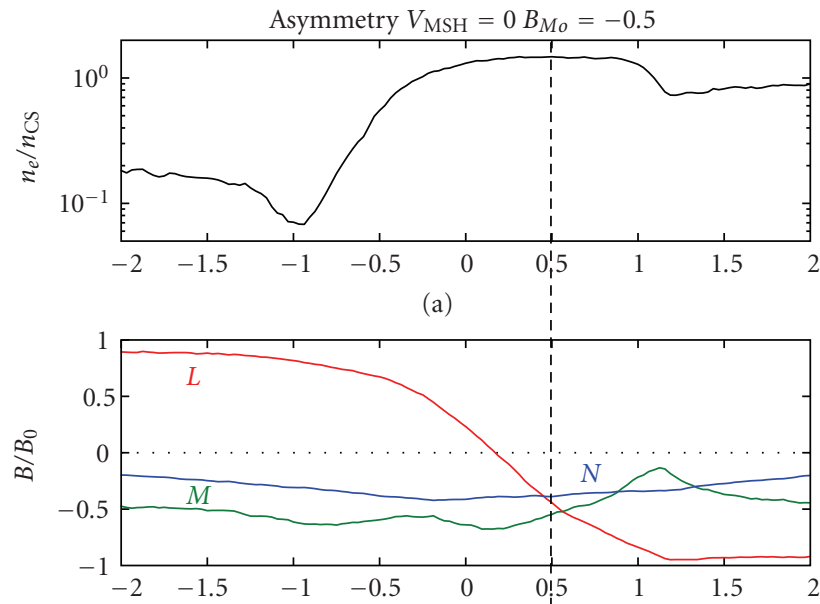

(b)

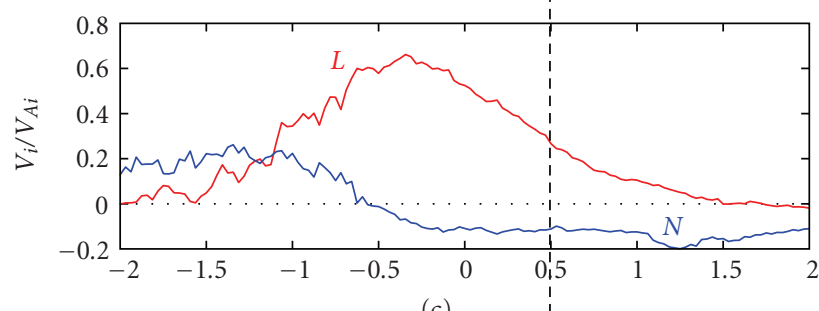

(c)

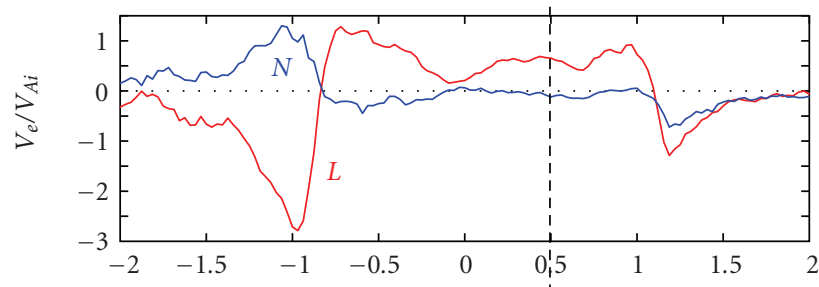

(d)

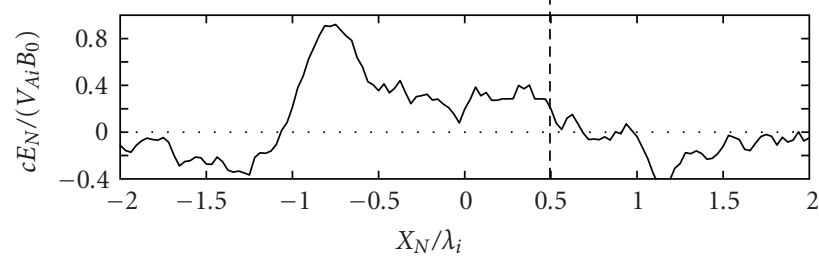

(e)

FIgURE 12: The cuts along the $N$-axis of the high-density structure at $L=4$. In the same format as Figure 5 except that ion and electron flows are now shown in Panels (c) and (d), respectively. The high-density peak (at $N=0.5$ ) is between the two density dips (at $N=-0.85$ and 1.27) and is on the magnetosheath side of the region showing $E_{N}>0$.

Figure $17(\mathrm{~b})$ compares $V_{L, \mathrm{XL}}$ with $V_{\mathrm{iL}, \mathrm{XL}}$ (solid circles) and with $V_{\text {eL,XL }}$ (open squares). The most eye-catching feature is the behavior of the $B_{M o}=0.5$ (red) cases. These have smaller $V_{L, \mathrm{XL}}$ but it is not necessarily because these cases have smaller $V_{\mathrm{iL}, \mathrm{XL}}$ compared to the others. Furthermore $V_{L, \mathrm{XL}}$ becomes negative as $V_{\mathrm{MSH}}$ is made smaller, but this happens even when $V_{\mathrm{iL}, \mathrm{XL}}$ stays positive. Meanwhile $V_{\mathrm{eL}, \mathrm{XL}}$ is negative as long as $B_{M o}=0.5$ (red open squares). These suggest that the role played by the electron flow at the X-line, 


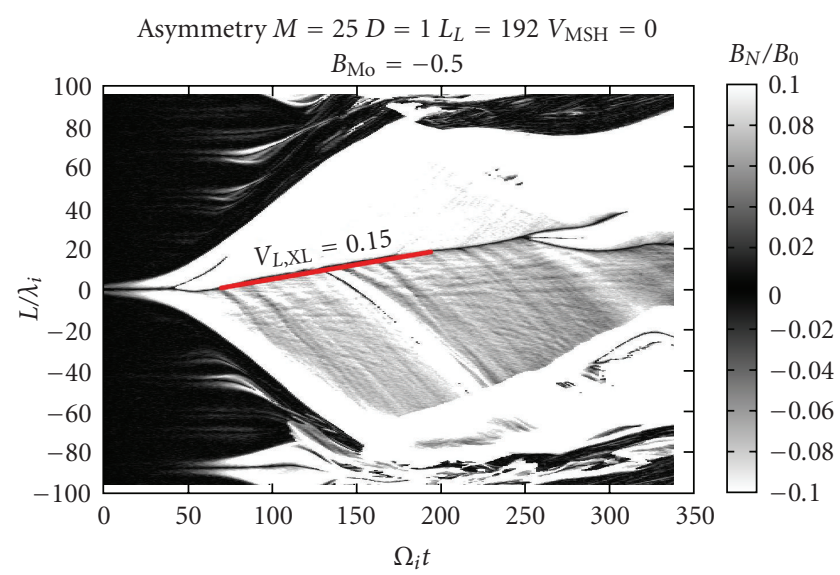

Figure 13: The same as Figure 9(b) but for the smaller mass ratio/larger simulation box case of $\left(V_{\mathrm{MSH}}, B_{M o}\right)=(0,-0.5)$. It shows that $V_{L, \mathrm{XL}}$ is constant for an extended time interval.

which is enhanced in the presence of nonzero guide field, is not limited to controlling the $\mathrm{X}$-line speed but can be as substantial as to determine its direction when the shear flow effects are weak.

Comparing the three cases having mostly the same X-line speed of $V_{L, \mathrm{XL}} \sim 0.15$ would be illuminating in this regard. The three cases are $\left(V_{\mathrm{MSH}}, B_{\mathrm{Mo}}\right)=(0,-0.5),(1,0)$, and $(1$, 0.5 ) (See Table 1). The X-line of the first case (blue symbols) has this speed even though the ion flow at the $\mathrm{X}$-line is pretty small. Instead, the electron flow is fast in the same direction (positive- $L$ ). The third case (red symbols) has almost the same X-line speed despite the considerably larger ion flow. It is noted that the electrons at the X-line are flowing fast in the opposite direction (negative- $L$ ). In the second case (black symbols) without guide field, there is only small difference between the ion and the electron flow at the X-line. The Xline speed coincides with the ion speed that is made nonzero by the slanted in-flow from the magnetosheath side.

\section{Discussion}

5.1. Summary. Table 2 summarizes all the results shown in this paper. The key issues would be as follows. (1) Magnetopause reconnection situation, namely, density asymmetry combined with shear flow and/or nonzero guide field, makes the X-line to slide along the current sheet. (2) In the presence of a shear flow, the ion flow at the X-line has nonzero $L$ component because the ion flow stagnation point is shifted towards the magnetospheric side by the density asymmetry and because the ion inflow from the magnetosheath side is slanted. When the shear is substantial, this drives the Xline to move in the same direction as the ion flow. (4) In the presence of nonzero guide field, even if the guide field is uniform initially, nonzero $L$-component of the current density is generated at the $\mathrm{X}$-line as reconnection develops. The current density is sustained by a fast electron flow at the X-line and, when the shear is weak, this can induce the $\mathrm{X}$-line to move in the same direction as the electron flow. (5) When the shear flow effects dominate, the X-line motion

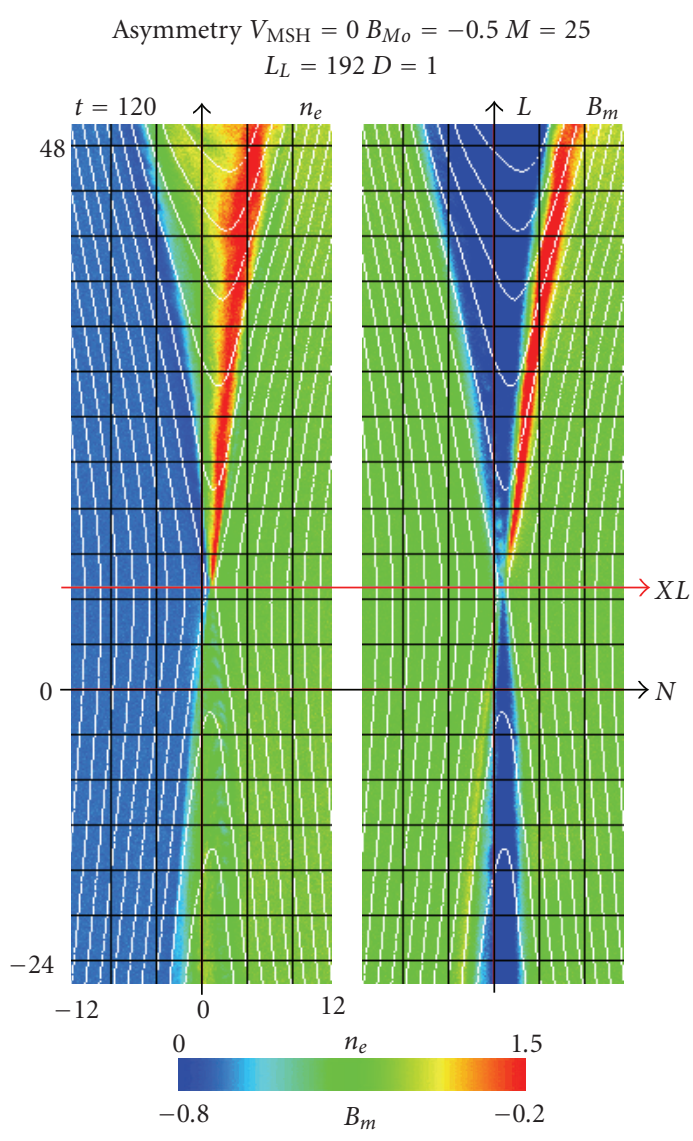

(a)

(b)

Figure 14: Two-dimensional contours of (a) density and (b) outof-plane magnetic field for the low mass ratio/larger simulation box case. The peculiar features in density and in $B_{M}$ seen in the upwarddirected jet are not local to the $\mathrm{X}$-line but are extended away from it.

can be opposite to the electron flow at the X-line. (6) When the shear flow effects are weakened, the guide field effects can dominate to move the X-line in the direction opposite to the magnetosheath flow. That is, in the close proximity of the subsolar region, an X-line may move opposite to the magnetosheath flow.

5.2. On the X-Line Motion. The results by Oka et al. [36] show the fact that the X-line motion can be in the opposite direction to that of the electron flow. It is not only the local physics at the $\mathrm{X}$-line that determines the $\mathrm{X}$-line motion, but also the mesoscale ion flow pattern surrounding the $\mathrm{X}$ line can be one of the determining factors. On the other hand, there can be cases where the local X-line physics can dominate. Indeed, the case studied by Swisdak et al. [24] is now understood by the case where the electron flow effects can easily dominate. The case studied has nonzero current density along the $L$-direction already in the initial condition and does not have the shear flow effects.

It would be observer friendly if we summarize our results in the magnetosphere-rest frame (the natural coordinates 


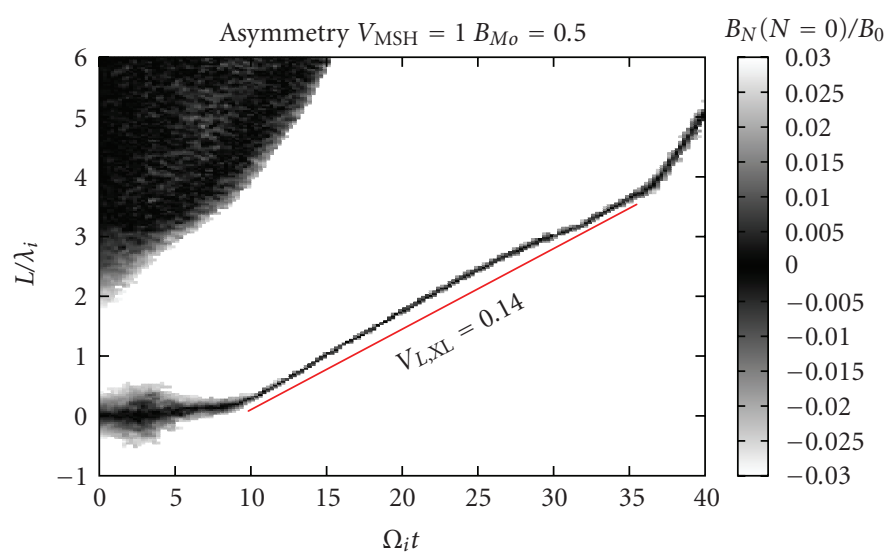

(a)

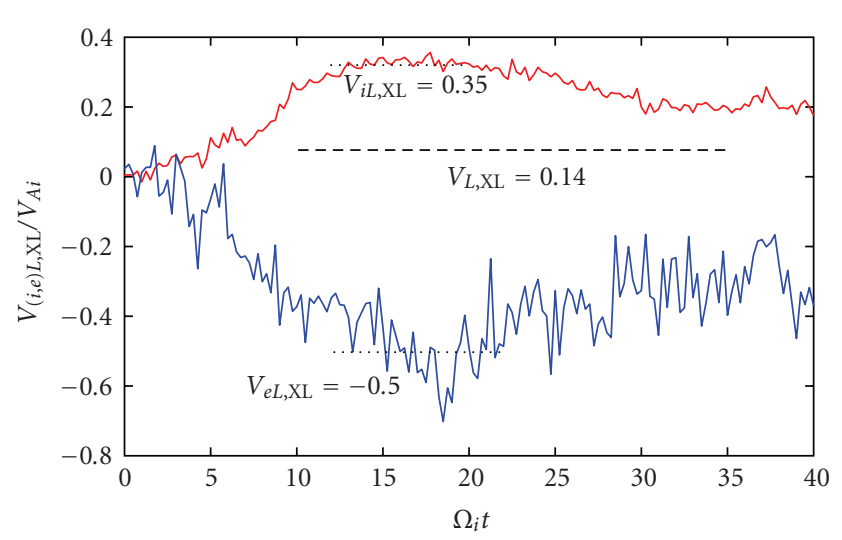

(b)

Figure 15: The results from the $\left(V_{\mathrm{MSH}}, B_{M o}\right)=(1,0.5)$ case, where the guide field and the velocity shear are considered to counteract in determining the direction of the X-line motion (See Table 1). The X-line motion shown in Panel (a) indicates that the shear flow effects dominate. Discussion on the X-line properties shown in Panel (b) given in the text, however, suggests that there are some roles played by the electrons as well.

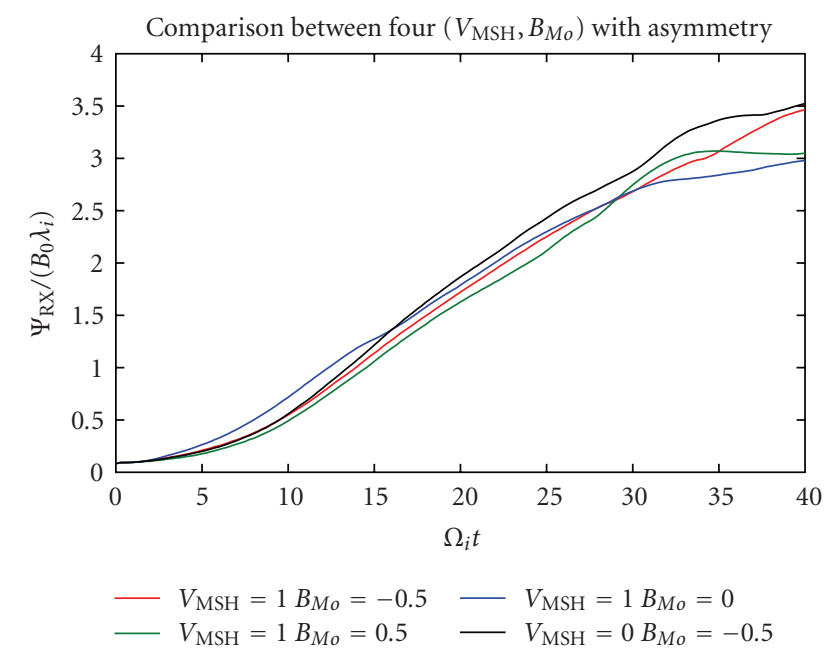

FIGURE 16: Growth curves of reconnection flux from various sets of $\left(V_{\mathrm{MSH}}, B_{M o}\right)$. There is little difference within the $\left(V_{\mathrm{MSH}}, B_{M o}\right)$ range surveyed.

for observers). This is done in Figure 18. Dealing the effects of guide field in terms of "error bars", one can say that the $\mathrm{X}$-line speed in the magnetosphere-rest frame is $0.73 V_{\mathrm{MSH}} \pm 0.15$, where $V_{\mathrm{MSH}}$ is the magnetosheath flow speed.

At this stage, it would be interesting to ask us how much interest there is in precisely determining the $\mathrm{X}$-line speed in the context of magnetopause reconnection. The magnetosheath flow seems to have the largest influence as soon as we leave the close proximity of the subsolar region. There $V_{\mathrm{XL}, \mathrm{MSP}} / V_{\mathrm{MSH}} \sim 0.7$ is what we obtain from our results.

5.3. Anomalous High-Density in the Jet. When the density asymmetry and the nonzero guide field are combined, we have found that one of the jets ejected out of the X-line has density higher than the magnetosheath value. The jet showing this anomalous feature is the one flowing in the same direction as the $\mathrm{X}$-line motion. We have also studied the effects of the shear flow on this anomalous density feature, and the results are as follows. (1) It is enhanced when the guide field counteracts against the flow in determining the $\mathrm{X}$-line motion and is seen in the jet flowing in the same direction as the one the guide field would have driven the Xline to move. That is, it is seen in the jet directed opposite the actual X-line motion if the flow effect dominates. (2) It is almost invisible when the guide field joins the flow in accelerating the X-line motion.

In an observer friendly manner, the anomalously high density would be easily detected (1) in the southward/stagnant jet in the northern hemisphere under duskto-dawn guide field or (2) in the northward/stagnant jet in the southern hemisphere under dawn-to-dusk guide field. While these imply a large chance for the anomalous jet to be detected, there is, to the best of the authors' knowledge, neither report in the literature, nor clear event in our own survey of the THEMIS data. We think this discrepancy has to do with the low magnetosheath plasma beta we assume in this study. It is 0.22 here but it is $>1$ in the real situation. Ions of a higher temperature will suffer less from the repelling electric field upon crossing the current sheet, which is the mechanism we propose to explain the anomalous density. While the plasma beta on the magnetosheath side here is rather low $(0.22)$, it is possible that more appropriate observational data may be obtained in the solar wind, where the averaged plasma beta is $\sim 0.25$ (e.g., [37]).

5.4. Limitations of the Present Study or Future Works. While we think there have been enough to learn from the present results, it is important to point out the limitations of the present study. The initial conditions used are limited such that they are close to the known Vlasov equilibrium. 


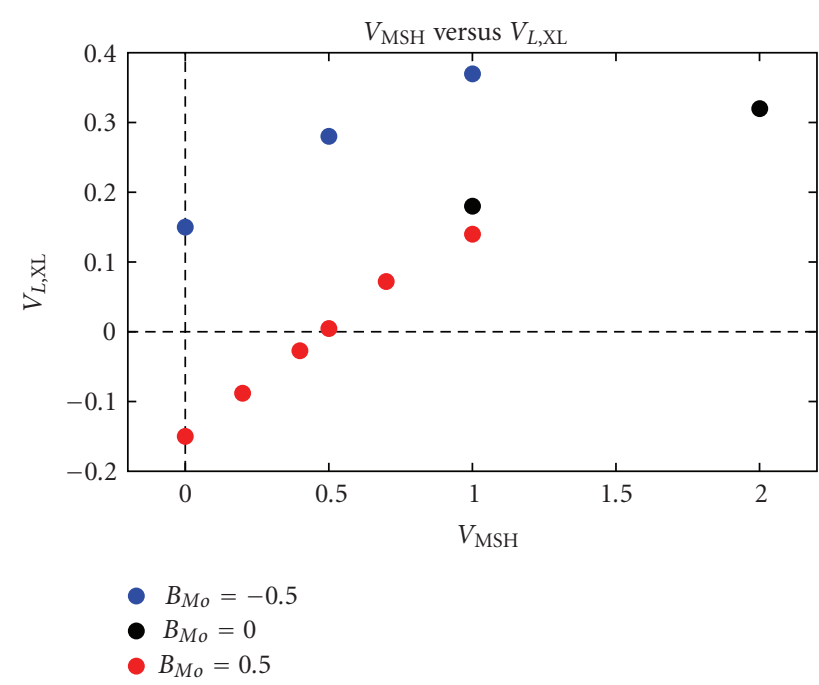

(a)

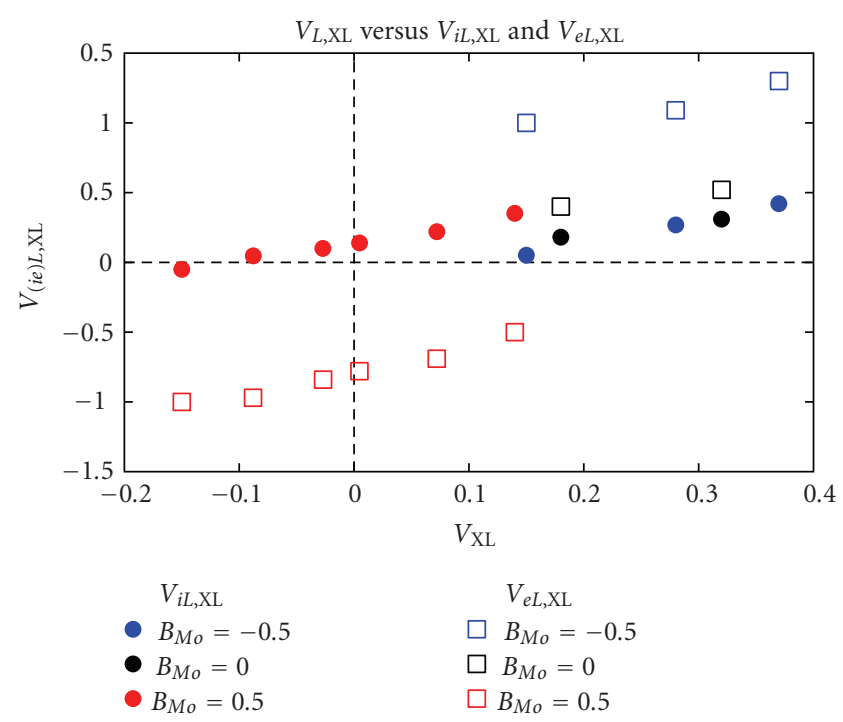

(b)

Figure 17: The summary plot for the discussion on the X-line motion. Different color indicates different $B_{M o}$ cases (blue, black, and red for $B_{M o}=-0.5,0$, and 0.5). (a) $V_{L, \mathrm{XL}}$ versus $V_{\mathrm{MSH}}$. While $V_{L, \mathrm{XL}}$ is larger for larger $V_{\mathrm{MSH}}$ in general, the X-line motion can be opposite to the magnetosheath flow when $V_{\mathrm{MSH}}$ is small and $B_{M o}=+0.5$. (b) Comparison of $V_{L, \mathrm{XL}}$ with $V_{\mathrm{iL}, \mathrm{XL}}$ (solid circles) and with $V_{\mathrm{eL}, \mathrm{XL}}$ (open squares). The interpretation is that the electron effects set in with the nonzero guide field and can dominate to determine the direction of the $\mathrm{X}$-line motion when the shear flow effects are small.

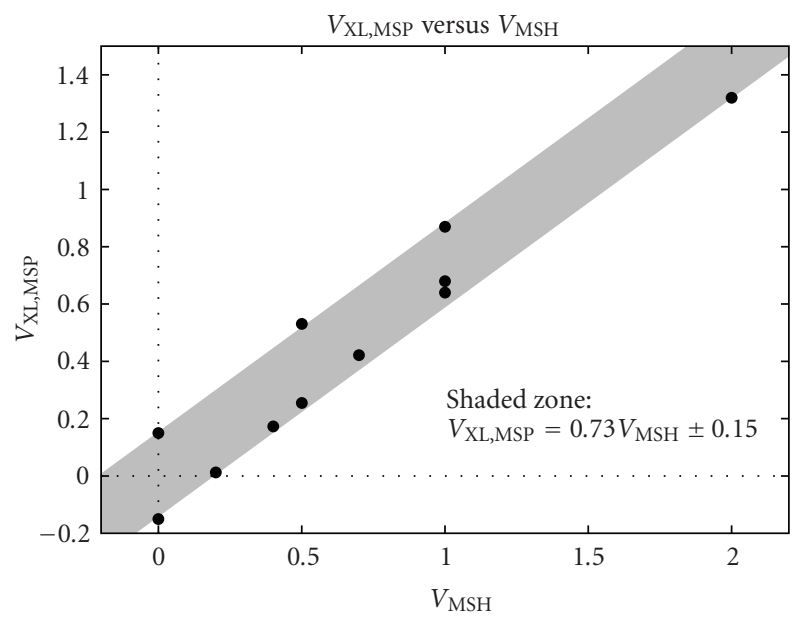

FIgUre 18: $V_{\mathrm{MSH}}$ versus $V_{L, \mathrm{XL}, \mathrm{MSP}}\left(V_{L, \mathrm{XL}}\right.$ in the magnetosphererest frame). The $\mathrm{X}$-line speed in the magnetosphere-rest frame is $0.73 V_{\mathrm{MSH}} \pm 0.15$ if the guide field effects are taken as "error bars."

The drawbacks are as follows. (1) The plasma beta in the magnetosheath is rather low. It seems to have led to the prediction of anomalous density in the jets that would not apply to the real magnetopause situation. (2) The guide field (the $M$-component) is assumed to be uniform initially. To have the guide field to vary across the magnetopause (i.e., $\partial B_{M} / \partial N$ is nonzero) is to set current density along the $L$ axis in the initial condition. This will enhance the electron effects in determining the direction of the X-line motion [24], and a larger area around the subsolar region may show X-lines moving opposite to the magnetosheath flow.
(3) The reconnecting magnetic field (the $L$-component) is symmetric across the current sheet. If the magnetic field intensity of this component is unbalanced, it will lead to differential convection speed along the $\mathrm{N}$-axis toward the Xline. This will lead to a different $\mathrm{X}$-line structure and there may well be a class of new effects arising. Aside from the Vlasov equilibrium issue, (4) the velocity shear is only in the $L$-component while in reality it is in the $M$-component as well. The effects of $V_{M}$ shear are left open. (5) 3D effects are out of the scope. As seen in numerous observations, the density gradient at the magnetopause excites Lower-Hybrid Drift Instability (LHDI) (e.g., $[38,39])$ and the coupling of this mode with reconnection has become a topic of intensive research $[17,19,40,41]$. It has already been pointed out that the density gradient even steepens at the X-line [27], which makes us expect that there is a direct coupling between the LHDI mode and the physics of the X-line. (6) Limited parameter range. It would have been more informative to the observers if we could show a cutoff in the maximum flow shear allowed. While Swisdak et al. [24] showed a clear prediction about a maximum allowed flow shear, our simulations did not find clear cutoff of reconnection activity. It might be because parameter range we surveyed was still limited. Extended parametric survey will be studied in a future work.

\section{Acknowledgments}

Numerical simulations are performed on NEC SX9 at JSS/JAXA. K. G. Tanaka was supported by Grant-in-Aid for Japan Society for the Promotion of Science (JSPS) Fellows (no. 20.9859). 


\section{References}

[1] R. L. Carovillano and J. M. Forbes, SOLAR-TERRESTRIAL PHYSICS, Principles and Theoretical Foundations, D. Reidel Publishing, Dordrecht, The Netherlands, 1983.

[2] B. Coppi and A. Ferrari, Plasmas in the Universe, IOS Press, Amsterdam, The Netherlands, 2000.

[3] E. Priest and T. Forbes, Magnetic Reconnection: MHD Theory and Applications, Cambridge University Press, New York, NY, USA, 2000.

[4] A. S. Sharma, R. Nakamura, A. Runov et al., "Transient and localized processes in the magnetotail: a review," Annales Geophysicae, vol. 26, no. 4, pp. 955-1006, 2008.

[5] G. Paschmann, B. U. Ö. Sonnerup, I. Papamastorakis et al., "Plasma acceleration at the Earth's magnetopause: evidence for reconnection," Nature, vol. 282, no. 5736, pp. 243-246, 1979.

[6] B. U. Ö Sonnerup, I. Papamastorakis, G. Paschmann, and H. Lühr, "Magnetopause properties from AMPTE/IRM observations of the convection electric field: method development," Journal of Geophysical Research A, vol. 82, no. 11, pp. 1213712159, 1987.

[7] J. T. Gosling, M. F. Thomsen, S. J. Bame, R. C. Elphic, and C. T. Russel, "Plasma flow reversals at the dayside magnetopause and the origin of asymmetric polar cap convection," Journal of Geophysical Research A, vol. 85, no. 6, pp. 8073-8084, 1990.

[8] A. Retinò, A. Vaivads, M. André et al., "Structure of the separatrix region close to a magmatic reconnection X-line: cluster observations," Geophysical Research Letters, vol. 33, no. 6, Article ID L06101, 4 pages, 2006.

[9] S. Eriksson, J. T. Gosling, T. D. Phan et al., "Asymmetric shear flow effects on magnetic field configuration within oppositely directed solar wind reconnection exhausts," Journal of Geophysical Research A, vol. 114, no. 7, Article ID A07103, 6 pages, 2009.

[10] J. T. Gosling, R. M. Skoug, D. J. McComas, and C. W. Smith, "Direct evidence for magnetic reconnection in the solar wind near 1 AU," Journal of Geophysical Research A, vol. 110, no. 1, Article ID A01107, 9 pages, 2005.

[11] J. Büchner, "Astrophysical reconnection and collisionless dissipation," Plasma Physics and Controlled Fusion, vol. 49, no. 12 B, pp. B325-B339, 2007.

[12] M. Swisdak, M. Opher, J. F. Drake, and F. Alouani Bibi, "The vector direction of the interstellar magnetic field outside the heliosphere," Astrophysical Journal, vol. 710, no. 2, pp. 17691775, 2010.

[13] J. E. Borovsky and M. Hesse, "The reconnection of magnetic fields between plasmas with different densities: scaling relations," Physics of Plasmas, vol. 14, no. 10, Article ID 102309, 10 pages, 2007.

[14] P. A. Cassak and M. A. Shay, "Scaling of asymmetric magnetic reconnection: general theory and collisional simulations," Physics of Plasmas, vol. 14, Article ID 102114, 2007.

[15] P. A. Cassak and M. A. Shay, "Structure of the dissipation region in fluid simulations of asymmetric magnetic reconnection," Physics of Plasmas, vol. 16, no. 5, Article ID 055704, 11 pages, 2009.

[16] E. G. Harris, "On a plasma sheath separating regions of oppositely directed magnetic field," Il Nuovo Cimento Series 10, vol. 23, no. 1, pp. 115-121, 1962.

[17] M. Scholer, I. Sidorenko, C. H. Jaroschek, R. A. Treumann, and A. Zeiler, "Onset of collisionless magnetic reconnection in thin current sheets: three-dimensional particle simulations," Physics of Plasmas, vol. 10, no. 9, pp. 3521-3527, 2003.
[18] P. L. Pritchett and F. V. Coroniti, "Three-dimensional collisionless magnetic reconnection in the presence of a guide field," Journal of Geophysical Research A, vol. 109, no. 1, Article ID A01220, 17 pages, 2004.

[19] P. Ricci, J. U. Brackbill, W. Daughton, and G. Lapenta, "Collisionless magnetic reconnection in the presence of a guide field," Physics of Plasmas, vol. 11, no. 8, pp. 4102-4114, 2004.

[20] M. Hesse, M. Kuznetsova, and J. Birn, "The role of electron heat flux in guide-field magnetic reconnection," Physics of Plasmas, vol. 11, no. 12, Article ID 5397, 11 pages, 2004.

[21] H. Karimabadi, W. Daughton, and K. B. Quest, "Physics of saturation of collisionless tearing mode as a function of guide field," Journal of Geophysical Research A, vol. 110, no. 3, Article ID A03214, 15 pages, 2005.

[22] H. Karimabadi, W. Daughton, and K. B. Quest, "Antiparallel versus component merging at the magnetopause: current bifurcation and intermittent reconnection," Journal of Geophysical Research A, vol. 110, no. 3, Article ID A03213, 22 pages, 2005.

[23] J. F. Drake, M. Swisdak, K. M. Schoeffler, B. N. Rogers, and S. Kobayashi, "Formation of secondary islands during magnetic reconnection," Geophysical Research Letters, vol. 33, no. 13, Article ID L13105, 4 pages, 2006.

[24] M. Swisdak, B. N. Rogers, J. F. Drake, and M. A. Shay, "Diamagnetic suppression of component magnetic reconnection at the magnetopause," Journal of Geophysical Research A, vol. 108, no. 5, Article ID 1218, 10 pages, 2003.

[25] P. L. Pritchett, "Collisionless magnetic reconnection in an asymmetric current sheet," Journal of Geophysical Research A, vol. 113, no. 6, Article ID A06210, 14 pages, 2008.

[26] P. L. Pritchett and F. S. Mozer, "Asymmetric magnetic reconnection in the presence of a guide field," Journal of Geophysical Research A, vol. 114, no. 1, Article ID A11210, 14 pages, 2009.

[27] K. G. Tanaka, A. Retinò, Y. Asano et al., "Effects on magnetic reconnection of a density asymmetry across the current sheet," Annales Geophysicae, vol. 26, no. 8, pp. 2471-2483, 2008.

[28] F. S. Mozer and P. L. Pritchett, "Regions associated with electron physics in asymmetric magnetic field reconnection," Geophysical Research Letters, vol. 36, no. 7, Article ID L07102, 5 pages, 2009.

[29] A. L. La Belle-Hamer, A. Otto, and L. C. Lee, "Magnetic reconnection in the presence of sheared flow and density asymmetry: applications to the Earth's magnetopause," Journal of Geophysical Research A, vol. 100, no. 7, pp. 11875-11889, 1995.

[30] K. G. Tanaka, I. Shinohara, and M. Fujimoto, "Effects of guide field on quick magnetic reconnection triggering," Geophysical Research Letters, vol. 31, no. 22, pp. 1-4, 2004.

[31] K. Haijima, K. G. Tanaka, M. Fujimoto, and I. Shinohara, "Electron temperature anisotropy effects on tearing mode in ion-scale current sheets," Advances in Space Research, vol. 41, no. 10, pp. 1643-1648, 2008.

[32] M. Øieroset, T. D. Phan, and M. Fujimoto, "Wind observations of asymmetric magnetic reconnection in the distant magnetotail," Geophysical Research Letters, vol. 31, no. 12, Article ID L12801, 4 pages, 2004.

[33] R. G. Kleva, J. F. Drake, and F. L. Waelbroeck, "Fast reconnection in high temperature plasmas," Physics of Plasmas, vol. 2, no. 1, pp. 23-34, 1995.

[34] B. N. Rogers, R. E. Denton, and J. F. Drake, "Signatures of collisionless magnetic reconnection," Journal of Geophysical Research A, vol. 108, no. 3, Article ID 1111, 7 pages, 2003. 
[35] J. F. Drake, M. A. Shay, W. Thongthai, and M. Swisdak, "Production of energetic electrons during magnetic reconnection," Physical Review Letters, vol. 94, no. 9, Article ID 095001, 4 pages, 2005.

[36] M. Oka, M. Fujimoto, T. K. M. Nakamura, I. Shinohara, and K.-I. Nishikawa, "Magnetic reconnection by a self-retreating X line," Physical Review Letters, vol. 101, no. 20, Article ID 205004, 4 pages, 2008.

[37] T. D. Phan, J. T. Gosling, and M. S. Davis, "Prevalence of extended reconnection X-lines in the solar wind at 1 AU," Geophysical Research Letters, vol. 36, no. 9, Article ID L09108, 5 pages, 2009.

[38] R. C. Davidson, N. T. Gladd, C. S. Wu, and J. D. Huba, "Effects of finite plasma beta on the lower-hybrid-drift instability," Physics of Fluids, vol. 20, no. 2, pp. 301-310, 1977.

[39] S. D. Bale, F. S. Mozer, and T. Phan, "Observation of lower hybrid drift instability in the diffusion region at a reconnecting magnetopause," Geophysical Research Letters, vol. 29, Article ID 2180, 4 pages, 2002.

[40] W. Daughton, G. Lapenta, and P. Ricci, "Nonlinear evolution of the lower-hybrid drift instability in a current sheet," Physical Review Letters, vol. 93, no. 10, Article ID 105004, 4 pages, 2004.

[41] K. G. Tanaka, I. Shinohara, and M. Fujimoto, "Quick magnetic reconnection triggering in an ion-scale current shet: transition from Type-I to Type-II," Geophysical Research Letters, vol. 32, no. 17, Article ID L17106, 4 pages, 2005. 

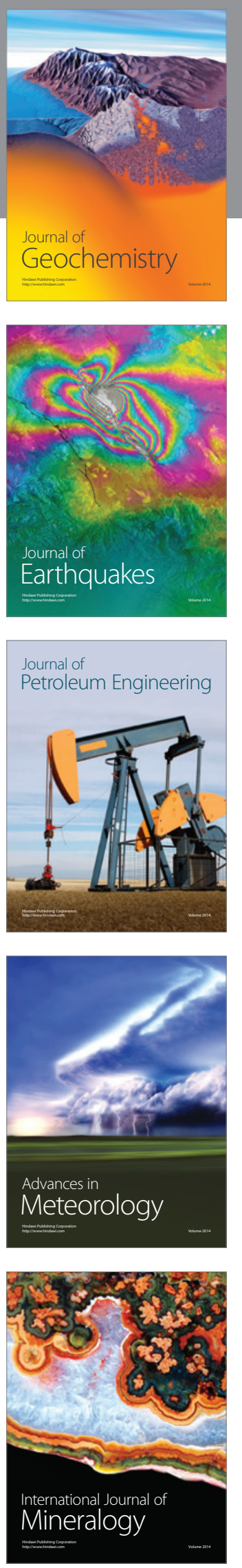
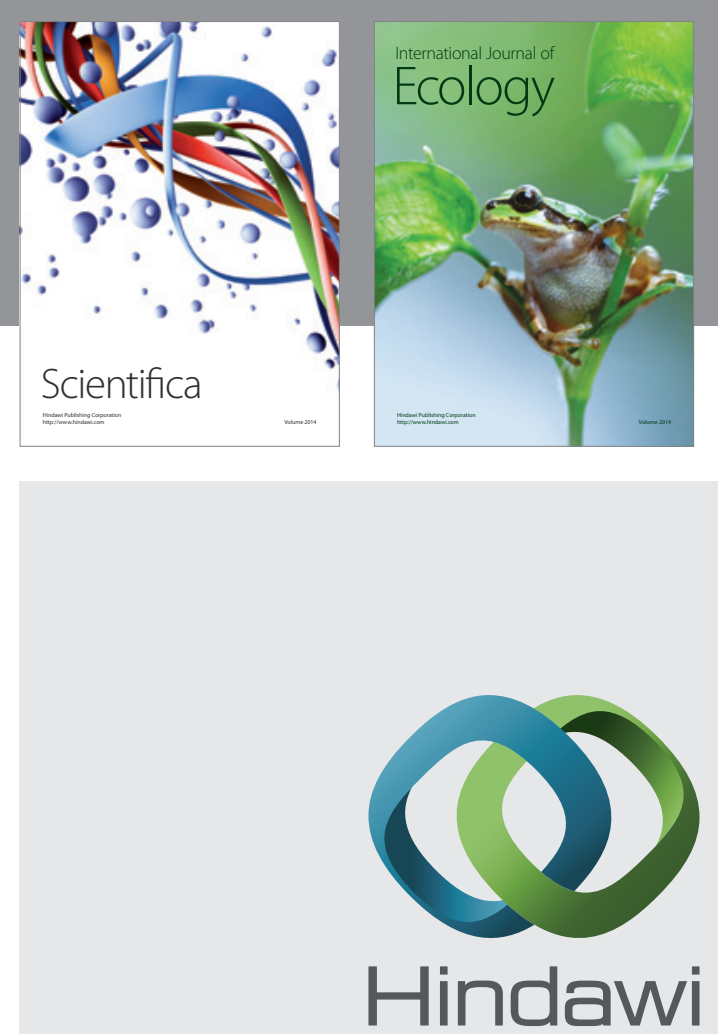

Submit your manuscripts at http://www.hindawi.com
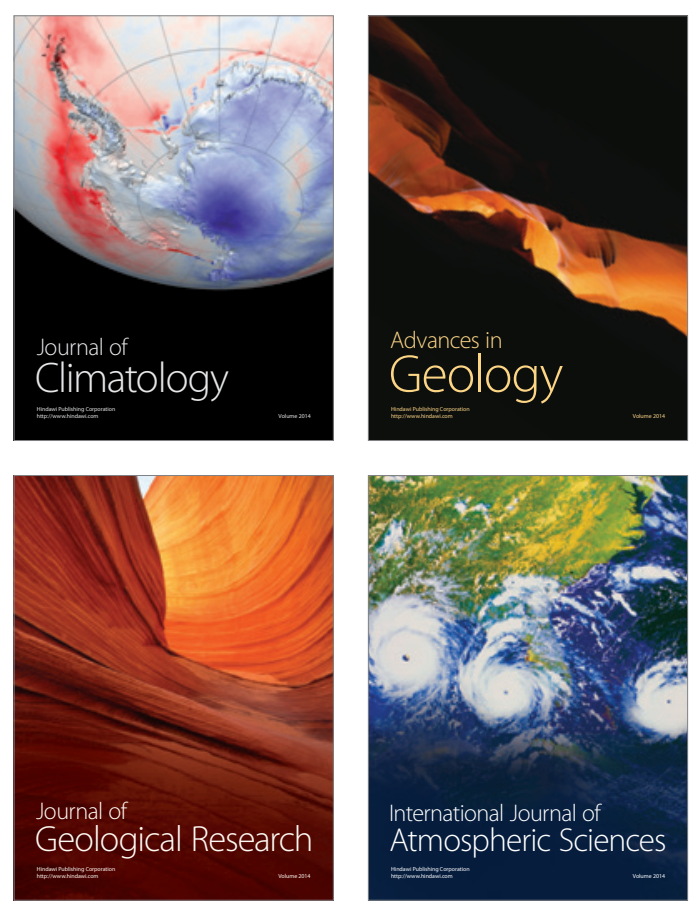
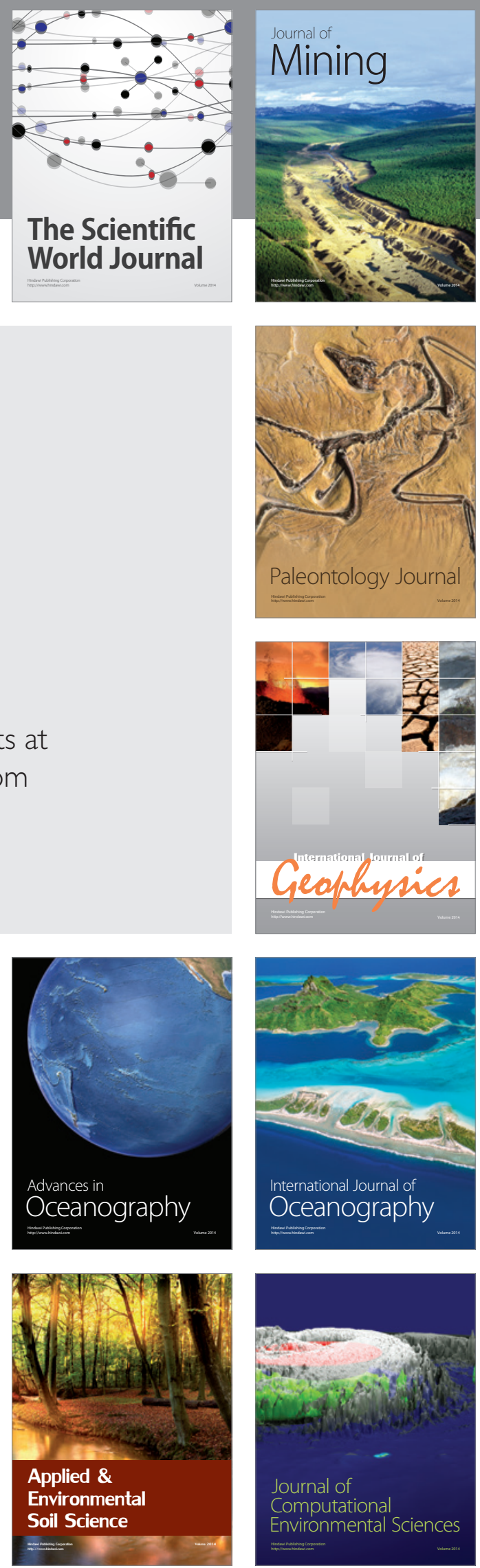